\title{
A new approach to Young measure theory, relaxation and convergence in energy
}

\author{
by
}

\author{
M.A. SYCHEV *
}

Sobolev Institute of Mathematics

Novosibirsk 630090, Russia

\begin{abstract}
The main idea of this paper is to reduce analysis of behavior of integral functionals along weakly convergent sequences to operations with Young measures generated by these sequences. We show that Young measures can be characterized as measurable functions with values in a special compact metric space and that these functions have a spectrum of properties sufficiently broad to realize this idea.

These new observations allow us to give simplified proofs of the results of gradient Young measure theory and to use them for deriving the results on relaxation and convergence in energy under optimal assumptions on integrands.

We think that this work helps to clarify role of Young measures. (C) 1999 L'Association Publications de l'Institut Henri Poincaré. Published by Elsevier B.V. All rights reserved
\end{abstract}

Key words: Integral functionals, Young measures, weak convergence.

AMS(MOS) Subject Classifications. 26B25, 35J20, 46E27, 46E35, 73C50.

RÉSUME. - L'idée principale de cet article est de ramener l'analyse du comportement de fonctionnelles intégrales portant sur des suites faiblement convergentes à des opérations sur les mesures de Young associées à ces suites. Nous montrons que les mesures de Young peuvent être caractérisées comme des fonctions mesurables à valeurs dans un certain espace métrique

\footnotetext{
* This research has been started during author's visit in Freiburgh University in 1995, carried out under the support of Russian Foundation for Basic Research, grant N 97-01-00508. The research was completed when the author held a fellowship in SISSA.

Annales de l'Institut Henri Poincaré - Analyse non linéaire - 0294-1449 
compact et que ces fonctions ont un ensemble de propriétés suffisament large pour mettre en œuvre cette idée.

Ces nouvelles observations nous permettent de donner des démonstrations simplifiées de résultats en théorie du gradient des mesures de Young et de les utiliser pour obtenir des résultats sur la relaxation et la convergence en énergie sous des hypothèses optimales sur les intégrandes.

Nous pensons aussi que ce travail aide à clarifier le rôle des mesures de Young.

(C) 1999 L'Association Publications de l'Institut Henri Poincaré. Published by Elsevier B.V. All rights reserved

\section{INTRODUCTION}

Consider the functional

$$
I(u)=\int_{\Omega} L(x, u(x), \nabla u(x)) d x,
$$

where $\Omega$ is a domain in $\mathbf{R}^{n}, u: \Omega \rightarrow \mathbf{R}^{m}$ and where $L: \Omega \times \mathbf{R}^{m} \times \mathbf{R}^{n m} \rightarrow$ $\mathbf{R}$ is a Caratheodory function. Some of the fundamental questions in the Calculus of Variations are

- under which conditions on $L$ is $I$ lower semicontinuous with respect to weak convergence in the Sobolev space $W^{1, p}\left(\Omega ; \mathbf{R}^{m}\right)$;

- can the lower semicontinuous envelope of $I$ be expressed as an integral functional;

- under which conditions do weak convergence $u_{k} \rightarrow u_{0}$ in $W^{1, p}\left(\Omega ; \mathbf{R}^{m}\right)$ and convergence in energy $I\left(u_{k}\right) \rightarrow I\left(u_{0}\right)$ imply strong convergence.

A number of results that answer these questions in different generality have been obtained in [1], [4], [14], [15], [19] since the fundamental work of Morrey [36] (see also [18], [38], [41], [44] for the scalar case $m=1$ ). In recent years it has become clear that optimal results are most easily stated and proved in the framework of Young measures ([3], [5], [29]-[30], [45]). The purpose of this paper is to present a streamlined and self-contained approach to the theory of gradient Young measures and its applications to the above problems.

We do not mention here all previous contributions in the area under discussion, but give appropriate references each time that we state a result similar to a known one or utilize a proof repeating a scheme discovered earlier somewhere else. 
In this paper we do not touch other problems in which Young measures play an essential role (see e.g. [8], [51]). An intensive study of the applications of Young measure theory to PDE started since the works of Tartar [49], [50].

We assume $\Omega$ is an open bounded subset of $\mathbf{R}^{n}$, with meas $(\partial \Omega)=0$, unless otherwise stated. By $W^{1, p}\left(\Omega ; \mathbf{R}^{m}\right)$ we denote the space of all measurable functions $u$ with finite norm $\|u\|_{W^{1, p}\left(\Omega ; \mathbf{R}^{m}\right)}:=\|u\|_{L^{p}\left(\Omega ; \mathbf{R}^{m}\right)}+$ $\|\nabla u\|_{L^{p}\left(\Omega ; \mathbf{R}^{n m}\right)}, \quad W_{0}^{1, p}\left(\Omega ; \mathbf{R}^{m}\right)$ is the closure of $C_{0}^{\infty}\left(\Omega ; \mathbf{R}^{m}\right)$ in $W^{1, p}\left(\Omega ; \mathbf{R}^{m}\right)$. By $W_{l o c}^{1, p}\left(\Omega ; \mathbf{R}^{m}\right)$ we denote the space of measurable mappings $u: \Omega \rightarrow \mathbf{R}^{m}$ such that $u \in W^{1, p}\left(\tilde{\Omega} ; \mathbf{R}^{m}\right)$ for any open set $\tilde{\Omega}$ compactly embedded in $\Omega(\tilde{\Omega} \subset \subset \Omega)$. The space $C_{0}\left(\mathbf{R}^{l}\right)$ is the closure of $C_{0}^{\infty}\left(\mathbf{R}^{l}\right)$ in the supremum norm. Equivalently,

$$
C_{0}\left(\mathbf{R}^{l}\right)=\left\{\Phi \in C\left(\mathbf{R}^{l}\right): \lim _{|v| \rightarrow \infty} \Phi(v)=0\right\} .
$$

Recall that $L(x, u, v): \Omega \times \mathbf{R}^{m} \times \mathbf{R}^{n m} \rightarrow \mathbf{R}$ is a Caratheodory integrand if $L(x, \cdot, \cdot)$ is continuous for a.a. $x$ and $L(\cdot, u, v)$ is measurable for all $u, v$. It is well known that $L$ is a Caratheodory integrand if and only if for each $\epsilon>0$ there exists a compact subset $\Omega_{c}$ of $\Omega$ such that meas $\left(\Omega \backslash \Omega_{\epsilon}\right) \leq \epsilon$ and the restriction of $L$ to $\Omega_{\epsilon} \times \mathbf{R}^{m} \times \mathbf{R}^{n m}$ is continuous, see [18].

From now on we will denote weak and strong convergence by $\rightarrow$ and $\rightarrow$, respectively. Convergence $f_{k} \rightarrow^{*} f$ in $L^{\infty}(\Omega)$ means convergence of integrals $\int_{\Omega} f_{k} g d x$ to $\int_{\Omega} f g d x$ for all $g \in L^{1}(\Omega)$. Convergence in $W_{\text {loc }}^{1, p}\left(\Omega ; \mathbf{R}^{m}\right)$ means convergence in $W^{1, p}\left(\tilde{\Omega} ; \mathbf{R}^{m}\right)$ for each $\tilde{\Omega} \subset \subset \Omega$.

The ball of radius $\epsilon$ with center at $x$ will be denoted by $B(x, \epsilon)$. We denote by $\mathbf{R}^{n m}$ the space of all $m \times n$ matrices and, for $A \in \mathbf{R}^{n m}$ and $x \in \mathbf{R}^{n}$ we denote by $A x$ the vector defined by matrix multiplication. By $l_{A}$ we denote a linear function $\mathbf{R}^{n} \rightarrow \mathbf{R}^{m}$ such that $l_{A}(x)=A x$ everywhere.

We write $\mathcal{M}\left(\mathbf{R}^{l}\right)$ for the space of all bounded Radon measures supported in $\mathbf{R}^{l}$, and $\|\mu\|_{\mathcal{M}}$ for the total variation of a measure $\mu$. To distinguish the action of a measure on a function from the scalar product we use the notation $\langle L ; \mu\rangle$ in the first case. Sometimes we also utilize more classical notation $\int L(v) d \mu$.

Recall the definition of Young measures

Definition 1.1. - A family $\left(\nu_{x}\right)_{x \in \Omega}$ of probability measures $\nu_{x} \in \mathcal{M}\left(\mathbf{R}^{l}\right)$ is called a Young measure if there exists a sequence of measurable functions $z_{k}: \Omega \rightarrow \mathbf{R}^{l}$ such that for each $\Phi \in C_{0}\left(\mathbf{R}^{l}\right)$

$$
\Phi\left(z_{k}\right) \rightarrow^{*} \bar{\Phi} \text { in } L^{\infty}(\Omega) \text {, where } \bar{\Phi}(x)=\left\langle\Phi ; \nu_{x}\right\rangle .
$$


If the $z_{k}$ are gradients of a sequence $u_{k} \in W^{1, p}\left(\Omega ; \mathbf{R}^{m}\right), p \in[1, \infty[$, which converges weakly in $W^{1, p}\left(\Omega ; \mathbf{R}^{m}\right)$ and for which the sequence $\left|\nabla u_{k}\right|^{p}$ is equi-integrable, then $\left(\nu_{x}\right)_{x \in \Omega}$ is called gradient p-Young measure.

Note that $u_{k}$ converges weakly in $W^{1, p}\left(\Omega ; \mathbf{R}^{m}\right)$ to some $u_{0}$, and $\nabla u_{0}(x)=\int_{\mathbf{R}^{n m}}(\cdot) d \nu_{x}$ for a.a. $x \in \Omega$ (cf. Theorem 3.7). Then $u_{0}$ is unique (up to additive constants) and is called underlying deformation.

We call a Young measure $\left(\nu_{x}\right)_{x \in \Omega}$ an homogeneous Young measure if $\nu_{x}$ does not depend on $x . G M_{p}(A)$ is the set of all homogeneous gradient p-Young measures with the center of mass at $A, G M_{\infty}(A)$ is the set of all homogeneous measures generated by gradients of sequences converging weakly* in $W^{1, \infty}\left(\Omega ; \mathbf{R}^{m}\right)$. We will frequently identify elements of the sets $G M_{p}(A), G M_{\infty}(A)$ with measures in $\mathcal{M}\left(\mathbf{R}^{n m}\right)$. Note that the sets $G M_{p}(A), G M_{\infty}(A)$ do not depend upon $\Omega$.

Note also that a function $\Phi\left(z_{0}\right): \Omega \rightarrow \mathbf{R}$ coincides with the function given by the action $\left\langle\Phi ; \delta_{z_{0}(\cdot)}\right\rangle$ of the family of Dirac masses $\delta_{z_{0}(\cdot)}$ on the function $\Phi$.

Other notations frequently used in this paper will be given in $\S 3$, which is completely devoted to general Young measure theory.

An improved version of fundamental theorem in Young measures by [3], [5] states that any sequence, which is bounded in $L^{p}$ with $p>0$, generates (after passage to a subsequence) a Young measure. Moreover, under certain conditions on an integrand the action of this measure on the integrand coincides with the limit of the values of the functional along the sequence (see [3], [5] and Theorem 3.7 stated below). In addition to these facts a characterization of the classes of Young measures generated by gradients of the Sobolev functions was obtained in [29].

These results give us a hope that analysis of behavior of integral functionals on weakly convergent sequences can be completely reduced to operations with Young measures generated by these sequences. In order to implement this idea one has to find a simple characterization of Young measures and effective tools for work with these objects. It turns out that such a characterization exists. In fact, Young measures are just measurable functions with values in a compact metric space with the metric having an integral representation (see Lemma 3.3). Although these functions are not so simple as the ones with values in $\mathbf{R}^{l}$, they still have a broad spectrum of properties (these properties are given by propositions 3.2-3.5).

It turns out that the characterization of Young measures as measurable functions and operations available for work with these functions suffice to prove all standard results of general Young measures theory. In this paper 
we do not discuss these matters, but concentrate on applications to gradient Young measure theory and problems of the Calculus of Variations related to behavior of integral functionals on weakly convergent sequences of Sobolev functions. In view of that we restrict our work to families of probability measures with measurable actions on continuous functions not proving explicitly that this class of families coincides with the class of Young measures. A curious reader can consult about the omitted issues in [46].

In $\S 2$ we will recall some auxiliary facts on Sobolev functions from [1], [7], [25]. The most important one is Theorem 2.1 recently proved in [30] (for an alternative proof see [23]).

In $\S 3$ we prove the characterization of Young measures as measurable functions into a special metric space and discuss basic properties of these functions: the Lusin property (Theorem 3.2), some quantitative estimates on how the convergence of the elements of the families of probability measures transforms into convergence of the families (Lemma 3.4), and a theorem on measurable selections (Theorem 3.5). We also state a version of the fundamental theorem in Young measures in a form convenient for our purposes (see Theorem 3.6). Since in this work we need to analyze behavior of integral functionals on sequences of Young measures, we extend the theorem on relation of the value of an integral functional on a Young measure with the values on a sequence of functions generating this measure to this generality (see Theorem 3.7).

In $\S 4$ we give simplified and self-contained proofs of the basic results of the theory of gradient Young measures (Theorems 4.2, 4.3), the main one of which is the classification of gradient p-Young measures (Theorem 4.3), obtained by Kinderlehrer \& Pedregal [29]. In the homogeneous case, we replace abstract duality arguments from [29] by ones relying on the integral formula for the metric corresponding to weak* convergence of measures and Theorem 3.7. Our arguments can be extended to the case of arbitrary integrands, see [47]. Then, we extend the result to the general case following construction proposed in $\S 6$ of [44].

In $\S 5$ we obtain some applications of the theory developed in $\S 2-4$ to the behavior of integral functionals on weakly convergent sequences of Sobolev functions. It turns out that the approach above leads to a simple proof of the relaxation theorem under optimal conditions on integrands if the standard growth conditions are assumed. 
Recall that

a function $L: \mathbf{R}^{n m} \rightarrow \mathbf{R}$ is called quasiconvex at $A \in \mathbf{R}^{n m}$ if for each $\phi \in C_{0}^{\infty}\left(\Omega ; \mathbf{R}^{m}\right)$ the inequality

$$
\int_{\Omega} L(A+\nabla \phi(y)) d y \geq L(A) \operatorname{meas} \Omega
$$

holds. This definition does not depend on the choice of an open set $\Omega$, with $\operatorname{meas}(\partial \Omega)=0(c f .[7])$.

THEOREM 1.2. - Let $L(x, u, v): \mathbf{R}^{n} \times \mathbf{R}^{m} \times \mathbf{R}^{n m} \rightarrow \mathbf{R}$ be a Caratheodory integrand such that

$$
A_{1}|v|^{p}+B_{1} \leq L(x, u, v) \leq A_{2}|v|^{p}+B_{2} \quad\left(p>1, \quad A_{2} \geq A_{1}>0\right) .
$$

Let

$$
\begin{gathered}
L^{q c}\left(x, u, v_{0}\right):=\inf _{\phi \in C_{0}^{\infty}\left(\Omega ; \mathbf{R}^{m}\right)} \frac{1}{\operatorname{meas} \Omega} \int_{\Omega} L\left(x, u, v_{0}+\nabla \phi(y)\right) d y \\
I^{q c}(u):=\int_{\Omega} L^{q c}(x, u(x), \nabla u(x)) d x .
\end{gathered}
$$

Then $L^{q c}$ satisfies the same estimates as $L$, is a Caratheodory integrand, and the function $v \rightarrow L^{q c}(x, u, v)$ is quasiconvex for a.a. $x \in \Omega$ and all $u \in \mathbf{R}^{m}$.

If $u_{k} \rightarrow u_{0}$ in $W^{1, p}\left(\Omega ; \mathbf{R}^{m}\right)$, then $\liminf _{k \rightarrow \infty} I\left(u_{k}\right) \geq I^{q c}\left(u_{0}\right)$. Moreover, there exists a sequence $u_{k} \in u_{0}+C_{0}^{\infty}\left(\Omega ; \mathbf{R}^{m}\right)$ such that the sequence $\left|\nabla u_{k}\right|^{p}$ is equi-integrable, $u_{k} \rightarrow u_{0}$ in $W^{1, p}\left(\Omega ; \mathbf{R}^{m}\right)$ and $I\left(u_{k}\right) \rightarrow I^{q c}\left(u_{0}\right)$.

Remarks.

1. If $L$ is continuous then $L^{q c}$ is continuous (see the proof of Theorem 1.2).

2. It follows from the second part of the theorem that $I^{q c}$ is the weak lower semicontinuous envelope of the original functional $I$.

The previous most significant results in relaxation (see [1], [10], [15]) involve some additional requirements on behavior of integrands with respect to $x, u$. In [1] a result on sequential weak lower semicontinuous envelope has been stated under less restrictive assumptions on growth of $L$. We will show how this result can be derived from Theorem 1.2 in the remark after the proof of the theorem. 
The following property of integral functionals is called the weak-strong convergence property:

$u_{k} \rightarrow u_{0}$ in $W^{1, p}\left(\Omega ; \mathbf{R}^{m}\right), I\left(u_{k}\right) \rightarrow I\left(u_{0}\right)$ imply $u_{k} \rightarrow u_{0}$ in $W^{1,1}\left(\Omega ; \mathbf{R}^{m}\right)$

We obtain precise characterization of integrands satisfying this property at a fixed function in $\S 5$ (see also [45]). Results of previous authors indicated some sufficient conditions for this property to hold at a function, everywhere (see [19], [29], [30], [41], [42], [52], [53] and papers cited in [44]). In the scalar case a pointwise characterization of this property has been obtained in [44].

Definition 1.3. (see [29], [30], [39]) - Let $L: \mathbf{R}^{n m} \rightarrow \mathbf{R}$ be continuous function such that $|L(v)| \leq A|v|^{p}+B$, $A>0$. Then $L$ is closed $p$ quasiconvex at $v_{0}$ if $\int_{\mathbf{R}^{n m}} L(\cdot) d \nu \geq L\left(v_{0}\right)$ for any homogeneous gradient p-Young measure $\nu$ (see Definition 1.1) with the center of mass at $v_{0} . L$ is strictly closed p-quasiconvex if the inequality is strict unless $\nu$ is a Dirac mass.

THEOREM 1.4. - Let $u_{0} \in W^{1, p}\left(\Omega ; \mathbf{R}^{m}\right)$ and let $L: \mathbf{R}^{n} \times \mathbf{R}^{m} \times \mathbf{R}^{n m} \rightarrow \mathbf{R}$ be a Caratheodory integrand satisfying the inequalities

$$
|L(x, u, v)| \leq A|v|^{p}+B, A>0, p>1
$$

Then the following assertions hold

1. If $L\left(x, u_{0}(x), v\right)$ is strictly closed p-quasiconvex at $v=\nabla u_{0}(x)$ for a.a. $x \in \Omega$, then the convergences $u_{k}-u_{0}$ in $W^{1, p}\left(\Omega ; \mathbf{R}^{m}\right), I\left(u_{k}\right) \rightarrow I\left(u_{0}\right)$ imply the convergence $u_{k} \rightarrow u_{0}$ in $W^{1,1}\left(\Omega ; \mathbf{R}^{m}\right)$ for any sequence $u_{k}$ such that the negative parts of $L\left(x, u_{k}(x), \nabla u_{k}(x)\right)$ are equi-integrable.

2. Conversely, if the convergences $u_{k} \rightarrow u_{0}$ in $W^{1, n}\left(\Omega ; \mathbf{R}^{m}\right)$ and $I\left(u_{k}\right) \rightarrow I\left(u_{0}\right)$ imply the convergence $u_{k} \rightarrow u_{0}$ in $W^{1,1}\left(\Omega ; \mathbf{R}^{m}\right)$ for all sequences $u_{k}$ such that $u_{k} \in u_{0}+C_{0}^{\infty}\left(\Omega ; \mathbf{R}^{m}\right)$ then either $L\left(x, u_{0}(x), v\right)$ or $-L\left(x, u_{0}(x), v\right)$ is strictly closed p-quasiconvex at $v=\nabla u_{0}(x)$ for a.a. $x \in \Omega$.

Remark. - In the situation in 1. the convergence

$$
L\left(x, u_{k}(x), \nabla u_{k}(x)\right) \rightarrow L\left(x, u_{0}(x), \nabla u_{0}(x)\right) \text { in } L^{1} \text { holds. }
$$

The "sufficient" part of the theorem has been proved in [30] through arguments introduced in [29]. We also will follow these arguments in the proof of this part of the theorem. 
Remark. - As proved in [45], for $p>1$ and integrands bounded from below strict closed p-quasiconvexity of $L$ at $v_{0}$ is equivalent to the property called in [45] strict p-quasiconvexity (see that paper for the motivations of this choice in terminology). The property is the following.

A function $L: \mathbf{R}^{n m} \rightarrow \mathbf{R}$ is strictly p-quasiconvex at $v_{0} \in \mathbf{R}^{n m}$ if $L$ is quasiconvex at $v_{0}$ and for every $c, \epsilon>0$ there exists $\delta=\delta(c, \epsilon)>0$ such that for $\phi \in C_{0}^{\infty}\left(\Omega ; \mathbf{R}^{m}\right)$ the inequalities

$$
\int_{\mathbf{R}^{n m}} L\left(v_{0}+\nabla \phi(x)\right) d x \leq\left(L\left(v_{0}\right)+\delta\right) \operatorname{meas} \Omega, \quad\|\nabla \phi\|_{L^{p}} \leq c
$$

imply the inequality

$$
\operatorname{meas}\{x \in \Omega:|\nabla \phi(x)|>\epsilon\}<\epsilon .
$$

Therefore, Theorem 1.4 indicates the additional requirement to quasiconvexity on behavior of integral functionals on linear functions both necessary and sufficient for the weak-strong convergence property to hold. In the scalar case $(m=1)$ strict p-quasiconvexity at a point $v_{0}$ becomes strict convexity: $\sum_{i} c_{i} L\left(v_{i}\right)>L\left(v_{0}\right)$ for any $c_{i} \geq 0, v_{i} \neq v_{0}$ such that $\sum c_{i}=1, \sum c_{i} v_{i}=v_{0}$ (see [44], [45]). In this case the second claim of Theorem 1.4 still holds if restricting considerations to the class of $u_{k}$ with equi-integrable $\left|\nabla u_{k}\right|^{p}$. We did not succeed to prove analogous result in the vector-valued case. As for the result from [45], it also has been obtained for the sequences $u_{k}$ with equi-integrable $\left|\nabla u_{k}\right|^{p}$, however assuming more restrictive growth conditions.

In this paper we do not treat the case $p=1$. We also do not consider the situation when the exponent of the Sobolev space, in which the weak convergence holds, is less than growth exponent of the integrands at infinity. For results in this direction and counterexamples see [2], [4], [6], [7] [9], [11], [16], [21], [22], [24], [32-35], and papers mentioned therein. It seems that the papers [6], [22] describe these results in the most generality.

\section{SOME AUXILIARY RESULTS}

This section contains some auxiliary facts utilized in this work. The basic fact from the theory of Sobolev functions which we need in this work is the following (see [1], [23], [25], [30,Th.3.10])

THEOREM 2.1. - Let $p \in] 1, \infty\left[\right.$ and let $u_{k}$ be a sequence bounded in $W^{1, p}\left(\Omega ; \mathbf{R}^{m}\right)$. There exists a subsequence $u_{j}$, and a sequence $v_{j} \in$ 
$W^{1, p}\left(\Omega ; \mathbf{R}^{m}\right)$ such that $\left|v_{j}-u_{j}\right|+\left|\nabla\left(v_{j}-u_{j}\right)\right| \rightarrow 0$ in measure and $\left|\nabla v_{j}\right|^{p}$ is equi-integrable.

It took a surprisingly long time to understand that the key point in some previous results is the above property of Sobolev functions. The technique sufficient to prove Theorem 2.1 had been utilized in [1] while the theorem was first stated explicitly only ten years later in [30,Th 3.10] (as a consequence of the stability result in the Hodge decomposition from [25]). An alternative proof has been proposed recently in [23, \$4].

Another important observation from [7] is the following fact. Let $\Omega$ be an open bounded set such that meas $(\partial \Omega)=0$ and $0 \in \Omega$. Let $\tilde{\Omega}$ be an arbitrary open and bounded set. Then by Vitali covering theorem $[56, \mathrm{Ch} .1]$ for any $\epsilon>0$ there exists a decomposition of $\tilde{\Omega}$ in sets of the form $a_{i}+\epsilon_{i} \bar{\Omega}$ $\left(i \in \mathbf{N}\right.$ ), where $\epsilon_{i}<\epsilon$, and a set $N_{0}$ of zero measure. Moreover

Lemma 2.2. - Let $u_{0} \in l_{A}+W_{0}^{1, p}\left(\Omega ; \mathbf{R}^{m}\right)$. For each $k \in \mathbf{N}$ consider the decomposition of $\tilde{\Omega}$ in disjoint sets of the type $a_{i}^{k}+\epsilon_{i}^{k} \Omega\left(i \in \mathbf{N}, \epsilon_{i}^{k} \leq 1 / k\right)$ and a set $N_{k}$ of null measure.

Defining $u_{k}$ as $A x+\epsilon_{i}^{k} u_{0}\left(\left(x-a_{i}^{k}\right) / \epsilon_{i}^{k}\right)$ for $x \in a_{i}^{k}+\epsilon_{i}^{k} \Omega$, and as $A x$ otherwise, we obtain that $u_{k} \rightarrow l_{A}$ in $W_{0}^{1, p}\left(\Omega ; \mathbf{R}^{m}\right)$ and, moreover, the sequence $\left|\nabla u_{k}\right|^{p}$ is equi-integrable with the same modulus of equi-integrability as the function $\left|\nabla u_{0}\right|^{p}$ multiplied by the factor (meas $\tilde{\Omega} /$ meds $\Omega$ ).

Proof. - is given in [7] with exception of the estimate for the modulus of equi-integrability of $\left|\nabla u_{k}\right|^{p}$ which follows immediately from the relation

$$
\begin{aligned}
& \operatorname{meas}\left\{x \in \tilde{\Omega}:\left|\nabla u_{k}(x)\right| \geq M\right\} / \operatorname{meas} \tilde{\Omega} \\
& \quad=\operatorname{meas}\left\{x \in \Omega:\left|\nabla u_{0}(x)\right| \geq M\right\} / \operatorname{meas} \Omega .
\end{aligned}
$$

Proposition 2.3. - Let $K \subset \mathbf{R}^{n}$ be a compact set of nonzero measure. Then, for each $\eta>0$ there exists an open set $O_{\eta}$ (consisting, possibly, of several domains) with smooth boundary and such that $\sup _{x \in O_{\eta}} \operatorname{dist}(x, K) \leq \eta$, meas $\left\{\left(K \backslash O_{\eta}\right) \cup\left(O_{\eta} \backslash K\right)\right\} \rightarrow 0$ as $\eta \rightarrow 0$.

Proof. - Let $f \geq 0$ be a usual mollifying kernel, i.e. let $f$ be smooth with the support in the unit ball and $\int_{\mathbf{R}^{n}} f=1$. Let $f_{\epsilon}(x)=\epsilon^{-n} f(x / \epsilon)$.

The convolution $f_{\epsilon} * \chi$, where $\chi$ is the characteristic function of $K$, is a smooth function with support lying in $2 \epsilon$-neighborhood of the support of $\chi$. Moreover, meas $\left(\operatorname{supp}\left(f_{\epsilon} * \chi\right) \backslash K\right) \rightarrow 0$ as $\epsilon \rightarrow 0$.

For almost all $\delta \in] 0,1\left[\right.$ the set $S_{\delta}^{\epsilon}=\left\{x: f_{\epsilon} * \chi=\delta\right\}$ is a smooth hypersurface (consisting, possibly, of several connected pieces). Actually, by the Sard theorem for a.a. $\delta \in] 0,1\left[\right.$ the inequality $\left|\nabla\left(f_{\epsilon} * \chi\right)(x)\right|>0$ holds 
for all $x \in S_{\delta}^{\epsilon}$. For each such $\delta$ and $x \in S_{\delta}^{\epsilon}$ the hypersurface $S_{\delta}^{\epsilon}$ is smooth in sufficiently small neighborhoods of $x$ by the implicit function theorem. Because of compactness of $S_{\delta}^{\epsilon}$ we obtain smoothness of $S_{\delta}^{\epsilon}$ everywhere.

Because of the convergence $f_{\epsilon} * \chi \rightarrow \chi$ a.e. in $\mathbf{R}^{n}$, for cach $\sigma>0$ we can isolate $\epsilon_{0}=\epsilon_{0}(\sigma)$ such that $\epsilon_{0} \leq \sigma$ and

$$
\operatorname{meas}\left\{x \in K:\left(f_{\epsilon_{0}} * \chi\right)(x) \geq 1-\sigma\right\} \geq \operatorname{meas} K-\sigma .
$$

The sets $O_{\eta}$ can be chosen as $\left\{x \in \mathbf{R}^{n}:\left(f_{\epsilon_{0}(\eta)} * \chi\right)(x)>1-\delta(\eta)\right\}$, where $\delta(\eta) \in] \eta, 2 \eta\left[\right.$ are such that the hypersurfaces $S_{\delta(\eta)}^{\epsilon_{0}(\eta)}$ are smooth. Indeed, by (2.1) meas $\left(K \backslash O_{\eta}\right) \leq \eta \rightarrow 0$ as $\eta \rightarrow 0$. The rest follows from the inclusion $O_{\eta} \subset \operatorname{supp}\left(f_{\epsilon_{0}(\eta)} * \chi\right)$.

The proof is completed.

\section{GENERAL YOUNG MEASURE THEORY. THEOREM ON BEHAVIOR OF INTEGRAL FUNCTIONALS ALONG SEQUENCES OF YOUNG MEASURES}

In this section we will prove that the families of Radon measures $\left(\nu_{x}\right)_{x \in \Omega}$ with measurable actions on elements of $C_{0}\left(\mathbf{R}^{l}\right)$ can be identified with measurable functions into a certain compact metric space (Lemma 3.3). This fact will allow us to apply some standard (but powerful) tools for constructing Young measurcs. In fact these tools, which are given by propositions 3.2-3.5, are enough to prove all standard results of Young measure theory (see [46]).

By the Riesz representation theorem, bounded linear functionals $l$ over the space $C_{0}\left(\mathbf{R}^{l}\right)$ are given by actions of Radon measures: $l(\Phi)=\langle\Phi ; \nu\rangle$. Therefore the space $\mathcal{M}\left(\mathbf{R}^{l}\right)$ of all Radon measures over $\mathbf{R}^{l}$ is dual to $C_{0}\left(\mathbf{R}^{l}\right)$. Moreover it is a Banach space with the total variation

$$
\|\nu\|_{\mathcal{M}}:=\sup _{\|\Phi\|_{C_{0}\left(\mathbf{R}^{l}\right) \leq 1}} \int_{\mathbf{R}^{l}} \Phi(v) d \nu
$$

as a norm.

Let $K_{c}=\left\{\nu \in \mathcal{M}\left(\mathbf{R}^{l}\right):\|\nu\|_{\mathcal{M}} \leq c\right\}$ and let $\left\{\Phi_{i}: i \in \mathbf{N}\right\}\left(\Phi_{i} \neq 0\right)$ be a dense set in $C_{0}\left(\mathbf{R}^{l}\right)$. The metric

$$
\rho(\nu, \mu)=\sum_{i=1}^{\infty} \frac{1}{2^{i}\left\|\Phi_{i}\right\|_{C}}\left|\left\langle\Phi_{i} ; \nu\right\rangle-\left\langle\Phi_{i} ; \mu\right\rangle\right|
$$


defined for elements of $\mathcal{M}\left(\mathbf{R}^{l}\right)$ endows $K_{c}$ with weak* topology for each $c>0$. It follows again from the Riesz representation theorem that $\left(K_{c}, \rho\right)$ is a compact metric space.

DEFINITION 3.1. - Let $\Omega$ be a bounded measurable subset of $\mathbf{R}^{n}$. A family of Radon measures $\left(\nu_{x}\right)_{x \in \Omega}$, where $\nu_{x} \in K_{c}$ for a.e. $x \in \Omega$, is called weak* measurable if for any $\Phi \in C_{0}\left(\mathbf{R}^{l}\right)$ the function $\left\langle\Phi ; \nu_{(\cdot)}\right\rangle$ is measurable. We denote the set of these families as $L_{w}\left(\Omega ; K_{c}\right)$.

A sequence $\left(\nu_{x}^{k}\right)_{x \in \Omega}$ of such families converges weakly* to a family $\left(\nu_{x}\right)_{x \in \Omega}$ in $L_{w}\left(\Omega ; K_{c}\right)$ if $\left\langle\Phi ; \nu_{(\cdot)}^{k}\right\rangle \rightarrow^{*}\left\langle\Phi ; \nu_{(\cdot)}\right\rangle$ in $L^{\infty}(\Omega), k \rightarrow \infty$, for each $\Phi \in C_{0}\left(\mathbf{R}^{l}\right)$. In this case we use the notation $\left(\nu_{x}^{k}\right)_{x \in \Omega} \rightarrow^{*}\left(\nu_{x}\right)_{x \in \Omega}$.

The space of weak* measurable families of Radon measures, which is $\cup_{c} L_{w}\left(\Omega ; K_{c}\right)$, is the dual space to $L^{1}\left(\Omega, C_{0}\left(\mathbf{R}^{l}\right)\right)$ (cf.[5],[17,p.588]) and this motivates our terminology.

In the following we frequently identify $\left(\nu_{x}\right)_{x \in \Omega}$ with the map $\nu: \Omega \rightarrow$ $\left(K_{r}, \rho\right)$ given by $\nu(x)=\nu_{x}$. It turns out that $\left(\nu_{x}\right)_{x \in \Omega}$ is weak ${ }^{*}$ measurable if and only if $\nu$ is measurable: for any closed subset $C$ of $\left(K_{c}, \rho\right)$ the set $\nu^{-1}(C)$ is measurable (see Lemma 3.3). This identification let us utilize some standard (but powerful) results on measurable maps, first of which is the Lusin property.

THEOREM 3.2. (Lusin type characterization of measurable functions) Let $\Omega$ be a bounded measurable subset of $\mathbf{R}^{n},(M, d)$ be a compact metric space. A function $\xi: \Omega \rightarrow(M, d)$ is measurable if and only if for any $\epsilon>0$ there exists a compact set $\Omega_{\epsilon} \subset \Omega$ such that meas $\left(\Omega \backslash \Omega_{\epsilon}\right) \leq \epsilon$ and the restriction of $\xi$ to $\Omega_{\epsilon}$ is continuous.

The proof is a straightforward modification of the proof of the standard version of this theorem.

LEMMA 3.3. - Let $\Omega$ be a bounded measurable subset of $\mathbf{R}^{n}$. Let $\nu_{x} \in K_{c}$ for a.a. $x \in \Omega$. The family $\left(\nu_{x}\right)_{x \in \Omega}$ is weak* measurable if and only if $\nu: \Omega \rightarrow\left(K_{c}, \rho\right)$ is a measurable mapping.

Proof. - Assume that a family $\left(\nu_{x}\right)_{x \in \Omega}$ has measurable actions on elements $\Phi$ of $C_{0}\left(\mathbf{R}^{l}\right)$. Let also $\left\{\Phi_{i}\right\} \subset C_{0}\left(\mathbf{R}^{l}\right)$ be a sequence of functions, which is dense in $C_{0}\left(\mathbf{R}^{l}\right)$. For given $\epsilon>0, i \in \mathbf{N}$ there exists a compact set $\Omega_{i} \subset \Omega$ such that the restriction of $\left\langle\Phi_{i} ; \nu_{(\cdot)}\right\rangle: \Omega \rightarrow \mathbf{R}$ to $\Omega_{i}$ is continuous and meas $\left(\Omega \backslash \Omega_{i}\right) \leq \epsilon / 2^{i}$. Then meas $\left(\Omega \backslash \cap \Omega_{i}\right) \leq \epsilon$ and the restrictions of all functions $\left\langle\Phi_{i} ; \nu_{(\cdot)}\right\rangle$ to $\cap \Omega_{i}$ are continuous. This implies continuity of the function $\nu: \cap \Omega_{i} \rightarrow\left(K_{c}, \rho\right)$. Then $\nu: \Omega \rightarrow\left(K_{c}, \rho\right)$ is a measurable function. 
Conversely, let $\nu$ be a measurable mapping from $\Omega$ into $\left(K_{c}, \rho\right)$. Then, by Theorem 3.2, the Lusin property holds for $\nu$. Thus, for any fixed $\epsilon>0$ there exists a compact subset $\Omega_{\epsilon}$ of $\Omega$ such that meas $\left(\Omega \backslash \Omega_{\epsilon}\right) \leq \epsilon$ and the restriction of $\nu$ to $\Omega_{\epsilon}$ is continuous in $\rho$-metric, that implies continuity of the restriction of the function $\left\langle\Phi ; \nu_{(\cdot)}\right\rangle$ to $\Omega_{\epsilon}$ for each $\Phi \in C_{0}\left(\mathbf{R}^{l}\right)$. Therefore $\left\langle\Phi ; \nu_{(\cdot)}\right\rangle$ is a measurable function for each $\Phi \in C_{0}\left(\mathbf{R}^{l}\right)$. This completes the proof. QED

We define the average $\operatorname{Av}\left(\mu_{x}\right)_{x \in \Omega}$ of a weak* measurable family of measures as follows

$$
\left\langle\Phi ; \operatorname{Av}\left(\mu_{x}\right)_{x \in \Omega}\right\rangle:=\frac{1}{\operatorname{meas} \Omega} \int_{\Omega}\left\langle\Phi ; \mu_{x}\right\rangle d x, \Phi \in C_{0}\left(\mathbf{R}^{l}\right) .
$$

It is clear that if $\mu_{x} \in K_{c}$ for a.e. $x \in \Omega$ then $\operatorname{Av}\left(\mu_{x}\right)_{x \in \Omega}$ is a linear functional over $C_{0}\left(\mathbf{R}^{l}\right)$ bounded in norm by $c$. Thus $\operatorname{Av}\left(\mu_{x}\right)_{x \in \Omega} \in K_{c}$.

We will need the following continuity property of the operation $\operatorname{Av}\left(\mu_{x}\right)_{x \in \Omega}$.

LEMMA 3.4. - Let $\Omega$ be a bounded measurable subset of $\mathbf{R}^{n}$ and let $\left(\mu_{x}^{1}\right)_{x \in \Omega},\left(\mu_{x}^{2}\right)_{x \in \Omega} \in L_{u}\left(\Omega, K_{c}\right)$. Then

1) If $\Omega_{\delta}$ is a measurable subset of $\Omega$ such that meas $\left(\Omega \backslash \Omega_{\delta}\right) \leq \delta$ meas $\Omega$ and $\rho\left(\mu_{x}^{1}, \mu_{x}^{2}\right) \leq \delta$ for all $x \in \Omega_{\delta}$, then

$$
\rho\left(\operatorname{Av}\left(\mu_{x}^{1}\right)_{x \in \Omega}, \operatorname{Av}\left(\mu_{x}^{2}\right)_{x \in \Omega}\right) \leq(2 c+1) \delta .
$$

2) If $\rho\left(\mu^{k}(\cdot), \mu(\cdot)\right) \rightarrow 0$ in measure, where $\left(\mu_{x}\right)_{x \in \Omega},\left(\mu_{x}^{k}\right)_{x \in \Omega} \in$ $L_{w}\left(\Omega ; K_{c}\right)$, then

$$
\left(\mu_{x}^{k}\right)_{x \in \Omega} \rightarrow^{*}\left(\mu_{x}\right)_{x \in \Omega} \text { in } L_{w}\left(\Omega ; K_{c}\right) \text { as } k \rightarrow \infty .
$$

Proof. - of the first claim is based on the representation formula for $\rho$. Actually, if $\Psi_{i}=\Phi_{i} /\left\|\Phi_{i}\right\|_{C\left(\mathbf{R}^{l}\right)}$ then

$$
\begin{gathered}
\rho\left(\operatorname{Av}\left(\mu_{x}^{1}\right)_{x \in \Omega}, \operatorname{Av}\left(\mu_{x}^{2}\right)_{x \in \Omega}\right)= \\
\sum_{i}\left\{\left|\int_{\mathbf{R}^{l}} \Psi_{i}(v) d \operatorname{Av}\left(\mu_{x}^{1}\right)_{x \in \Omega}-\int_{\mathbf{R}^{l}} \Psi_{i}(v) d \operatorname{Av}\left(\mu_{x}^{2}\right)_{x \in \Omega}\right| / 2^{i}\right\}= \\
\sum_{i}\left\{\left|\int_{\Omega} \int_{\mathbf{R}^{l}} \Psi_{i}(v) d \mu_{x}^{1} d x-\int_{\Omega} \int_{\mathbf{R}^{l}} \Psi_{i}(v) d \mu_{x}^{2} d x\right| / 2^{i} \text { meas } \Omega\right\} \leq \\
\sum_{i}\left\{\left(\int_{\Omega}\left|\int_{\mathbf{R}^{l}} \Psi_{i}(v) d \mu_{x}^{1}-\int_{\mathbf{R}^{l}} \Psi_{i}(v) d \mu_{x}^{2}\right| d x\right) /\left(2^{i} \text { meas } \Omega\right)\right\} \leq
\end{gathered}
$$




$$
\begin{gathered}
2 c \delta+\sum_{i}\left\{\left(\int_{\Omega_{\delta}}\left|\int_{\mathbf{R}^{l}} \Psi_{i}(v) d \mu_{x}^{1}-\int_{\mathbf{R}^{l}} \Psi_{i}(v) d \mu_{x}^{2}\right| d x\right) /\left(2^{i} \text { meas } \Omega\right)\right\}= \\
2 c \delta+\int_{\Omega_{\delta}}\left\{\sum_{i}\left|\int_{\mathbf{R}^{l}} \Psi_{i}(v) d \mu_{x}^{1}-\int_{\mathbf{R}^{l}} \Psi_{i}(v) d \mu_{x}^{2}\right| / 2^{i}\right\} d x /(\operatorname{meas} \Omega) \leq \\
2 c \delta+\int_{\Omega_{\delta}} \rho\left(\mu_{x}^{1}, \mu_{x}^{2}\right) d x / \operatorname{meas} \Omega \leq 2 c \delta+\delta
\end{gathered}
$$

The second assertion of the theorem is an immediate consequence of the first one. Actually, let $\tilde{\Omega}$ be a measurable subset of $\Omega$. By the first statement of the theorem $\operatorname{Av}\left(\mu_{x}^{k}\right)_{x \in \tilde{\Omega}} \rightarrow^{*} \operatorname{Av}\left(\mu_{x}\right)_{x \in \tilde{\Omega}}$ as $k \rightarrow \infty$. Hence, for any $\Phi \in C_{0}\left(\mathbf{R}^{l}\right)$ the convergence $\left\langle\Phi ; \mu_{(\cdot)}^{k}\right\rangle \rightarrow^{*}\left\langle\Phi ; \mu_{(\cdot)}\right\rangle$ in $L^{\infty}(\Omega)$ holds.

The proof of Lemma 3.4 is completed. QED

We recall also a version of the theorem on measurable selections from [31] (for more sophisticated versions of such theorems see [12]).

Let $\Omega$ be a bounded measurable subset of $\mathbf{R}^{n}$ and let $(M, d)$ be a compact metric space. A mapping $V: \Omega \rightarrow 2^{M}$ is a closed measurable multi-valued mapping if, for a.a. $x \subset \Omega$, the set $V(x)$ is closed and if for any closed subset $C$ of $M$ the set $\{x \in \Omega: V(x) \cap C \neq \emptyset\}$ is measurable.

THEOREM 3.5. - If $V: \Omega \rightarrow 2^{M}$ is a closed measurable multivalued mapping then there exists a measurable selection, i.e. a measurable map $\nu: \Omega \rightarrow(M, d)$ such that $\nu(x) \in V(x)$ for a.a. $x \in \Omega$.

The following result is a version of fundamental theorem of Young measures (see [3], [5], [20], [49], [50], [54], [55]) stating weak* compactness of families of Radon measures.

THEOREM 3.6. (Compactness result) - Let $\Omega$ be a measurable bounded subset of $\mathbf{R}^{n}$ and let $\left(\nu_{x}^{k}\right)_{x \in \Omega} \in L_{w}\left(\Omega ; K_{c}\right)$. Then there exists a subsequence $\left(\nu_{x}^{k}\right)_{x \in \Omega}$ (not relabeled) and $a\left(\nu_{x}\right)_{x \in \Omega} \in L_{w}\left(\Omega ; K_{c}\right)$, such that

$$
\left(\nu_{x}^{k}\right)_{x \in \Omega} \rightarrow^{*}\left(\nu_{x}\right)_{x \in \Omega} \text { in } L_{w}\left(\Omega ; K_{c}\right)
$$

that is $\left\langle\Phi ; \nu_{(\cdot)}^{k}\right\rangle \rightarrow^{*}\left\langle\Phi ; \nu_{(\cdot)}\right\rangle$ in $L^{\infty}(\Omega)$ for any $\Phi \in C_{0}\left(\mathbf{R}^{l}\right)$.

If $\left(\nu_{x}^{k}\right)_{x \in \Omega}$ is a family of probability measures then $\left(\nu_{x}\right)_{x \in \Omega}$ also consists of probability measures provided there exists a function $g: \mathbf{R}^{l} \rightarrow \mathbf{R}^{+}$such that $\lim _{|v| \rightarrow \infty} g(v)=\infty$ and

$$
\int_{\Omega} \int_{\mathbf{R}^{i}} g(v) d \nu_{x}^{j} d x \leq c .
$$

Vol. $16, n^{\circ}$ 6-1999. 
In particular, each sequence of measurable functions $z^{j}: \Omega \rightarrow \mathbf{R}^{l}$ such that $\int_{\Omega} g\left(z^{j}(x)\right) d x \leq c$ contains a subsequence generating a Young measure.

Proof. - of the compactness result is given in [5], [20], [30]. It follows from the duality $L^{\infty}\left(\Omega ; \mathcal{M}\left(\mathbf{R}^{l}\right)\right)$ and $L^{1}\left(\Omega ; C_{0}\left(\mathbf{R}^{l}\right)\right)$ and the BanachAlaouglu theorem. For a proof in context of the concept of Young measures as measurable functions see [46].

To prove the second part of the theorem note that $\nu_{x}$ is nonnegative for a.a. $x \in \Omega$ and $\left\|\nu_{x}\right\|_{\mathcal{M}} \leq 1$.

Let $\Omega_{k}$ be an increasing sequence of compact subsets of $\Omega$ such that meas $\left(\Omega \backslash \Omega_{k}\right) \rightarrow 0$ as $k \rightarrow \infty$ and the restrictions of $\nu: \Omega \rightarrow\left(K_{1}, \rho\right)$ to $\Omega_{k}$ are continuous. Let $i \in \mathbf{N}$ and $\Omega_{i, k}=\left\{x \in \Omega_{k}:\left\|\nu_{x}\right\|_{\mathcal{M}} \leq 1-1 / i\right\}$. Then $\Omega_{i, k}$ is a closed subset of $\Omega_{k}$. Suppose that meas $\Omega_{i, k}>0$.

Consider $\nu^{j}=\operatorname{Av}\left(\nu_{x}^{j}\right)_{x \in \Omega_{i, k}}, \nu=\operatorname{Av}\left(\nu_{x}\right)_{x \in \Omega_{i, k}}$. By the assumptions we have $\nu^{j} \rightarrow^{*} \nu$ (this follows from the convergence $\left(\nu_{x}^{k}\right)_{x \in \Omega} \rightarrow^{*}\left(\nu_{x}\right)_{x \in \Omega}$ in $\left.L_{w}\left(\Omega ; K_{c}\right)\right), \int_{\mathbf{R}^{\prime}} g(v) d \nu^{j} \leq c$, and $\|\nu\|_{\mathcal{M}} \leq 1-1 / i$. In particular one has, for all $C<\infty$ the inequality

$$
\nu^{j}\left(\mathbf{R}^{l} \backslash B(0, C)\right) \inf \{g(v):|v| \geq C\} \leq c
$$

holds. Thus for any sufficiently large $C$ we have $\nu^{j}(B(0, C)) \geq 1-1 / 2 i$ for all $j \in \mathbf{N}$.

If $\Phi: \mathbf{R}^{l} \rightarrow[0,1]$ is continuous, $\Phi(v)=1$ for $|v| \leq C$, and $\Phi(v)=0$ for $|v| \geq 2 C$, then

$$
\int_{\mathbf{R}^{i}} \Phi(v) d \nu^{j} \geq 1-1 / 2 i>1-1 / i \geq \int_{\mathbf{R}^{l}} \Phi(v) d \nu
$$

for all $j \in \mathbf{N}$. This contradiction with the convergence $\nu^{j} \rightarrow^{*} \nu$ proves that meas $\Omega_{i, k}=0$. Thus meas $\left(\cup_{i, k} \Omega_{i, k}\right)=0$ and, as a consequence, $\left\|\nu_{x}\right\|_{\mathcal{M}}=1$ for a.e. $x \in \Omega$. QED

Recall that the main idea of this work is to replace analysis of behavior of integral functionals along weakly convergent sequences by work with Young measures generated by these sequences. In order to implement this idea we need to characterize the cases when the action of a Young measure on an integrand coincides with the limit of the values assumed by the integral functional at a sequence generating this measure. In the general case only the lower semicontinuity result holds (see [3], [43], and Theorem 3.7). Since the inner demands of the theory which we develop in this paper require work with sequences of Young measures instead of functions, we have also to indicate such a characterization in this, more general, situation. 
It turns out that a relevant characterization is the following one.

Let $F(x, v): \mathbf{R}^{n} \times \mathbf{R}^{l} \rightarrow \mathbf{R}^{+}$be a nonnegative Caratheodory integrand, $\left(\nu_{x}^{i}\right)_{x \in \Omega} \in L_{w}\left(\Omega ; K_{c}\right)(i \in \mathbf{N})$. We will say that $\left(\nu_{x}^{i}\right)_{x \in \Omega}$ satisfies the tightness condition with the integrand $F$ on $\Omega \subset \mathbf{R}^{n}$ if

$$
\lim _{M \rightarrow \infty} \sup _{i} \int_{\Omega}\left(\int_{\mathbf{R}^{i}} \xi_{M}(F(x, \cdot)) d \nu_{x}^{i}\right) d x=0
$$

where $\xi_{M}: \mathbf{R} \rightarrow \mathbf{R}$ is a continuous function satisfying the requirements: $0 \leq \xi_{M}(t) \leq t$ everywhere, $\xi_{M}(t)=0$ for $t<M, \xi_{M}(t)=t$ for $t \geq 2 M$.

It is easy to see that in the case $\nu_{(\cdot)}^{i}=\delta_{z_{i}(\cdot)}$ the tightness condition coincides with equi-integrability of the sequence $F\left(\cdot, z_{i}(\cdot)\right)$.

The next theorem gives answer to the above question.

THEOREM 3.7. - Let $\Omega$ be a bounded measurable subset of $\mathbf{R}^{n}$.

Let $\left(\nu_{x}^{i}\right)_{x \in \Omega}$ be a sequence of families of probability measures with support in $\mathbf{R}^{l}$, and let $L(x, v): \Omega \times \mathbf{R}^{l} \rightarrow \mathbf{R}$ be a Caratheodory integrand. Suppose that $\left(\nu_{x}^{i}\right)_{x \in \Omega}$ satisfies the tightness conditions with the negative part $L^{-}$of $L$ (therefore the integrals of the functions $\int_{\mathbf{R}^{l}} L(\cdot, v) d \nu_{(\cdot)}^{i}$ are either finite or equal $+\infty)$ and that $\left(\nu_{x}^{i}\right)_{x \in \Omega}$ generates a family of probability measures $\left(\nu_{x}\right)_{x \in \Omega}$.

Then

$$
\liminf _{i \rightarrow \infty} \int_{\Omega}\left(\int_{\mathbf{R}^{i}} L(x, v) d \nu_{x}^{i}\right) d x \geq \int_{\Omega}\left(\int_{\mathbf{K}^{i}} L(x, v) d \nu_{x}\right) d x .
$$

Moreover, $\lim _{i \rightarrow \infty} \int_{\Omega} \int_{\mathbf{R}^{l}} L(x, v) d \nu_{x}^{i} d x \rightarrow \int_{\Omega} \int_{\mathbf{R}^{l}} L(x, v) d \nu_{x} d x$ if and only if $\left(\nu_{x}\right)_{x \in \Omega}$ satisfies the tightness condition with $|L|$. In this case $\int_{\mathbf{R}^{l}} L(\cdot, v) d \nu_{(\cdot)}^{i} \rightarrow \int_{\mathbf{R}^{l}} L(\cdot, v) d \nu_{(\cdot)}$ in $L^{1}$.

Proof. - Let $\Omega_{k}$ be a sequence of compact sets such that meas $\left(\Omega \backslash \Omega_{k}\right) \rightarrow$ 0 as $k \rightarrow \infty$ and the restrictions of $L$ to $\Omega_{k} \times \mathbf{R}^{l}$ are continuous. Let us prove first the theorem under the additional requirement of boundedness of $L$ from below.

Consider a sequence of continuous functions $\Phi_{j}: \mathbf{R}^{l} \rightarrow[0,1]$ such that $\Phi_{j}(v)=1$ for $v \in B(0, j), \Phi_{j}(v)=0$ for $v \in \mathbf{R}^{l} \backslash B(0,2 j)$, and $\Phi_{j}(v)$ is nondecreasing in $j$ for any fixed $v \in \mathbf{R}^{l}$.

For fixed $k \in \mathbf{N}, j \in \mathbf{N}$ let $w$ be a modulus of continuity of the restriction of $L$ to $\Omega_{k} \times[-2 j, 2 j]^{l}$. Decompose $\Omega_{k}$ on the sets $K_{1}, \ldots, K_{m}$ in such a Vol. $16, \mathrm{n}^{\circ}$ 6-1999. 
way that $\operatorname{diam} K_{p} \leq \delta, p \in\{1, \ldots, m\}$. Let also $x_{p} \in K_{p}, p=1, \ldots, m$. Then, for any fixed $p \in\{1, \ldots, m\}$

$$
\begin{gathered}
\int_{\mathbf{R}^{l}} \Phi_{j}(v) L\left(x_{p}, v\right) d \nu_{(\cdot)}^{i} \longrightarrow^{*} \int_{\mathbf{R}^{\prime}} \Phi_{j}(v) L\left(x_{p}, v\right) d \nu_{(\cdot)} \text { in } L^{\infty} \text { as } i \rightarrow \infty \\
\left|\Phi_{j}(v)\right|\left|L\left(x_{p}, v\right)-L(y, v)\right| \leq w(\delta), \text { if } y \in K_{p}, v \in \mathbf{R}^{\prime}
\end{gathered}
$$

Thus, letting $\delta \rightarrow 0$ we obtain

$$
\int_{\mathbf{R}^{l}} \Phi_{j}(v) L(\cdot, v) d \nu_{(\cdot)}^{i} \rightarrow^{*} \int_{\mathbf{R}^{\prime}} \Phi_{j}(v) L(\cdot, v) d \nu_{(\cdot)} \text { in } L^{\infty}\left(\Omega_{k}\right), i \rightarrow \infty .
$$

Since $L$ is bounded from below the Fatou lemma implies

$$
\int_{\Omega_{k}}\left(\int_{\mathbf{R}^{l}} \Phi_{j}(v) L(x, v) d \nu_{x}^{i}\right) d x \rightarrow \int_{\Omega_{k}}\left(\int_{\mathbf{R}^{l}} L(x, v) d \nu_{x}^{i}\right) d x
$$

as $j \rightarrow \infty, i \in \mathbf{N}$ (the same holds for the family $\left.\left(\nu_{x}\right)_{x \in \Omega}\right)$. Thus

$$
\liminf _{i \rightarrow \infty} \int_{\Omega_{k}}\left(\int_{\mathbf{R}^{l}} L(x, v) d \nu_{x}^{i}\right) d x \geq \int_{\Omega_{k}}\left(\int_{\mathbf{R}^{l}} L(x, v) d \nu_{x}\right) d x .
$$

It is also clear that for the complete convergence we need tightness of $\left(\nu_{x}^{i}\right)_{x \in \Omega_{k}}$ with $L^{+}$. In this case $\int_{\mathbf{R}^{\prime}} L(\cdot, v) d \nu_{(\cdot)}^{i} \rightarrow \int_{\mathbf{R}^{l}} L(\cdot, v) d \nu_{(\cdot)}$ in $L^{1}\left(\Omega_{k}\right)$.

Because meas $\left(\Omega \backslash \Omega_{k}\right) \rightarrow 0$ as $k \rightarrow \infty$ and $L$ is bounded from below the desired result follows. Theorem 3.7 is thus proved for integrands bounded from below. For general integrands consider the auxiliary integrands $L^{n}=\max \{L,-n\}$ for which the inequality

$$
\liminf _{j \rightarrow \infty} \int_{\Omega}\left(\int_{\mathbf{R}^{\prime}} L^{n}(x, v) d \nu_{x}^{i}\right) d x \geq \int_{\Omega}\left(\int_{\mathbf{R}^{\prime}} L^{n}(x, v) d \nu_{x}\right) d x
$$

has been proved. In view of the tightness condition for $\left(\nu_{x}^{i}\right)_{x \in \Omega}$ with $L^{-}$ and Fatou's lemma the same holds for the original integrand. Moreover we have complete convergence if and only if $\left(\nu_{x}^{i}\right)_{x \in \Omega}$ satisfies the tightness condition with $L^{+}$, and in this case $\int_{\mathbf{R}^{\prime}} L(\cdot, v) d \nu_{(\cdot)}^{i} \rightarrow \int_{\mathbf{R}^{\prime}} L(\cdot, v) d \nu_{(\cdot)}$ in $L^{1}$ as $i \rightarrow \infty$.

The proof of the theorem is completed. QED

In the following we will frequently use Proposition 3.8 . 
Proposition 3.8. - Let $\Omega$ be a bounded measurable subset of $\mathbf{R}^{n}$. Let $z_{j}: \Omega \rightarrow \mathbf{R}^{l}$ be a sequence of measurable functions that generates a Young measure $\left(\nu_{x}\right)_{x \in \Omega}$. Then the following assertions hold.

1.) The sequence $z_{j}$ converges in measure if and only if $\nu_{x}$ is a Dirac mass for a.a. $x \in \Omega$.

2.) If the sequence $y_{j}$ satisfies $z_{j}-y_{j} \rightarrow 0$ in measure as $j \rightarrow \infty$ then it generates the same Young measure.

Proof. - Convergence $z_{j} \rightarrow z_{0}$ in measure implies strong convergence $\Phi\left(z_{j}\right) \rightarrow \Phi\left(z_{0}\right)$ in $L^{1}(\Omega)$ for each $\Phi \in C_{0}\left(\mathbf{R}^{l}\right)$. Hence $z_{j}$ generates the family $\delta_{z_{0}(\cdot)}: \Phi\left(z_{j}\right) \rightarrow^{*}\left\langle\Phi ; \delta_{z_{0}(\cdot)}\right\rangle=\Phi\left(z_{0}\right)$ in $L^{\infty}$ for all $\Phi \in C_{0}\left(R^{l}\right)$.

To prove the inverse note that given $\epsilon>0$ we can find a bounded continuous integrand $L: \Omega \times \mathbf{R}^{l} \rightarrow \mathbf{R}$ and a set $\Omega_{\epsilon} \subset \Omega$ such that $\operatorname{meas}\left(\Omega \backslash \Omega_{\epsilon}\right)<\epsilon, 0 \leq L \leq 1$ everywhere, and for each $x \in \Omega_{\epsilon}$ we have $L(x, v)=1$ for $v \in B\left(z_{0}(x), \epsilon / 2\right), L(x, v)=0$ for $v \notin \in B\left(z_{0}(x), \epsilon\right)$. By Theorem 3.7 we have $L\left(\cdot, z_{j}(\cdot)\right)-^{*} L\left(\cdot, z_{0}(\cdot)\right)$ in $L^{\infty}(\Omega)$. Since $\int_{\Omega} L\left(x, z_{0}(x)\right) d x \geq \operatorname{meas} \Omega_{\epsilon}$ we infer

$$
\lim _{j}: \infty \operatorname{meas}\left\{x \in \Omega: z_{j}(x) \in B\left(z_{0}, \epsilon\right)\right\} \geq \operatorname{meas} \Omega_{\epsilon} \geq \operatorname{meas} \Omega-\epsilon .
$$

This implies convergence $z_{j} \rightarrow z_{0}$ in measure.

Proof of the second part of Proposition 3.8 is immediate since $\Phi\left(y_{j}\right)-\Phi\left(z_{j}\right) \rightarrow 0$ in $L^{1}(\Omega)$ for each $\Phi \in C_{0}\left(\mathbf{R}^{l}\right)$. QED

\section{GRADIENT YOUNG MEASURE THEORY}

Recall first the definition of gradient p-Young measures. Let $\Omega$ be an open bounded domain with meas $(\partial \Omega)=0$.

Definition 4.1. - A Young measure $\left(\nu_{x}\right)_{x \in \Omega}$ is a gradient p-Young measure, $p \in\left[1, \infty\left[\right.\right.$, if it is generated by gradients $\nabla u_{j}$ of a sequence $u_{j} \in W^{1, p}\left(\Omega ; \mathbf{R}^{m}\right)$ such that $u_{j}$ converges weakly in $W^{1, p}\left(\Omega ; \mathbf{R}^{m}\right)$ and the functions $\left|\nabla u_{j}\right|^{p}$ are equi-integrable.

If $u_{j}-u_{0}$ in $W^{1, p}\left(\Omega ; \mathbf{R}^{m}\right)$, then Theorem 3.7 implies $\nabla u_{0}(x)=$ $\int_{\mathbf{R}^{n m}}(\cdot) d \nu_{x}$ for a.a. $x \in \Omega$. The function $u_{0}$ is called the underlying deformation.

A Young measure $\left(\nu_{x}\right)_{x \in \Omega}$ is called homogeneous if it does not depend on $x$. By $G M_{p}(A)$ we denote the set of all homogeneous gradient $\mathrm{p}$-Young measures with the center of mass at $A$. By $G M_{\infty}(A)$ we denote those of 
them which are generated by gradients of sequences converging weakly* in $W^{1, \infty}\left(\Omega ; \mathbf{R}^{m}\right)$. Both these sets do not depend on $\Omega$.

By $\nu \diamond A$ we denote the measure obtained by exchanging the center of mass of $\nu$ to $A$ : if $\nu$ is generated as a gradient p-Young measure by $\nabla u_{k}$ and $B$ is the center of mass of $\nu$ then $\nu \diamond A$ is generated by the sequence $\nabla u_{k}+l_{A-B}$.

The main purpose of this section is to give self-contained proofs of the following two basic results of gradient Young measure theory first proved in [27]-[29].

THEOREM 4.2. - Let $\left(\nu_{x}\right)_{x \in \Omega}$ be a gradient $p$-Young measure with underlying deformation $u_{0}, p \in[1, \infty[$. Then

1) (Averaging principle) If there exists an $A \in \mathbf{R}^{n m}$ such that $u_{0}-l_{A} \in$ $W_{0}^{1, p}\left(\Omega ; \mathbf{R}^{m}\right)$ then $\operatorname{Av}\left(\nu_{x}\right)_{x \in \Omega} \in G M_{p}(A)$. If $u_{0}-l_{A} \in W_{0}^{1, \infty}\left(\Omega ; \mathbf{R}^{m}\right)$ then $\operatorname{Av}\left(\delta_{\nabla u_{0}(x)}\right)_{x \in \Omega} \in G M_{\infty}(A)$.

2) (Localization principle) For a.a. $x \in \Omega$ the measure $\nu_{x}$ is a homogeneous gradient p-Young measure.

Corollary of Theorem 4.2. - Let $L: \mathbf{R}^{n m} \rightarrow \mathbf{R}$ be continuous, $|L(v)| \leq A|v|^{p}+B, p \in[1, \infty[$. Then

1. the following identities hold

$\inf _{\nu \in G M_{p}(A)}\langle L ; \nu\rangle=\inf _{\nu \in G M_{\infty}(A)}\langle L ; \nu\rangle=\inf _{\phi \in C_{0}^{\infty}\left(\Omega ; \mathbf{R}^{m}\right)} \frac{1}{\operatorname{meas} \Omega} \int L(A+\nabla \phi(x)) d x ;$

2. the function $L$ is quasiconvex at $A$ if and only if

$$
\inf _{\nu \in G M_{p}(A)}\langle L ; \nu\rangle \geq L(A)
$$

THEOREM 4.3. (Characterization of gradient p-Young measures) $-A$ family $\left(\nu_{x}\right)_{x \in \Omega} \in L_{w}\left(\Omega ; K_{1}\right)$ of probability measures is a gradient $p$-Young measure with $p \in[1, \infty[$ if and only if

(i) there exists $u_{0} \in W^{1, p}\left(\Omega ; \mathbf{R}^{m}\right)$ such that $\int_{\mathbf{R}^{n m}}(\cdot) \nu_{x}=\nabla u_{0}(x)$ for a.a. $x \in \Omega$;

(ii) for a.a. $x \in \Omega$ the inequality $L\left(\nabla u_{0}(x)\right) \leq \int_{\mathbf{R}^{n m}} L(v) d \nu_{x}$ holds for any quasiconvex function $L$ such that $c \leq L(v) \leq A|v|^{p}+B$.

(iii) $\int_{\Omega} \int_{\mathbf{R}^{n m}}\left(1+|v|^{p}\right) d \nu_{x} d x<\infty$.

Remark. - The theorem asserts that a probability measure $\nu \in \mathcal{M}\left(\mathbf{R}^{n m}\right)$ is a homogeneous gradient $\mathrm{p}$-Young measure if and only if $\left\langle 1+|\cdot|^{p} ; \nu\right\rangle<\infty$ and $L(\langle\cdot ; \nu\rangle) \leq\langle L ; \nu\rangle$ for all quasiconvex $L$ with $c \leq L \leq A|\cdot|^{p}+B$. 
Thus $\left(\nu_{x}\right)_{x \in \Omega} \in L_{w}\left(\Omega ; K_{1}\right)$ is gradient $\mathbf{p}$-Young measure if and only if (i), (iii) hold and for a.a. $x \in \Omega$ the measure $\nu_{x}$ is a homogeneous gradient p-Young measure.

In the scalar case $\min \{n, m\}=1$ quasiconvexity becomes convexity. Therefore any family of probability measures satisfying conditions (i),(iii) is a gradient p-Young measure. This fact was also implicitly proved in $[44, \S 6]$ through approximation results from [18, Ch.10].

To prove Theorems $4.2,4.3$ we will need two simple auxiliary propositions

PROPOSITION 4.4. - 1. Let $p \in] 1, \infty\left[,\left(\nu_{x}\right)_{x \in \Omega}\right.$ be a Young measure generated by gradients of a sequence $u_{j}$ bounded in $W^{1, p}\left(\Omega ; \mathbf{R}^{m}\right.$ ) (no assumptions on equi-integrability of $\left|\nabla u_{k}\right|^{p}$ ), and let $u_{0}$ be the underlying deformation. Then $\left(\nu_{x}\right)_{x \in \Omega}$ is generated also by gradients of a sequence $v_{k} \in u_{0}+C_{0}^{\infty}\left(\Omega ; \mathbf{R}^{m}\right)$ such that the functions $\left|\nabla v_{k}\right|^{p}$ are equi-integrable and $v_{k} \rightarrow u_{0}$ in $W^{1, p}\left(\Omega ; \mathbf{R}^{m}\right)$. In particular, $\left(\nu_{x}\right)_{\boldsymbol{x} \in \Omega}$ is a gradient $p$-Young measure.

2. Let $p \in\left[1, \infty\left[\right.\right.$, let $\left|\nabla u_{j}\right|^{p}$ be equi-integrable and $u_{j} \rightarrow u_{0} \in$ $W^{1, p}\left(\Omega ; \mathbf{R}^{m}\right)$ in $W_{\text {loc }}^{1, p}\left(\Omega ; \mathbf{R}^{m}\right)$. Let also $\nabla u_{j}$ generate a Young measure $\left(\nu_{x}\right)_{x \in \Omega}$. Then there exists a sequence $v_{k} \in u_{0}+C_{0}^{\infty}\left(\Omega ; \mathbf{R}^{m}\right)$ gradients of which generate $\left(\nu_{x}\right)_{x \in \Omega}$ as a gradient $p$-Young measure.

It is clear that in the case $p>1$ the statement of the second assertion is close to the statement of the first one, but proofs of the seconds parts of both propositions 4.4 and 4.5 do not involve Theorem 2.1. We state the second assertions of these propositions separately in order to show that, like in [29], Theorem 4.3 can be proved without using Theorem 2.1.

Note that Proposition 4.4 was proved in [29] using arguments similar to those in [1] and Theorem 4.3. Theorem 2.1, which we use in the proofs of the first parts of Propositions 4.4 and 4.5, were established later in [30].

Proof. - 1. By Theorem 2.1 there exists a subsequence $u_{j}$ (not relabeled) and a sequence $w_{j} \in W^{1, p}\left(\Omega ; \mathbf{R}^{m}\right)$ such that $\left|\nabla w_{j}\right|^{p}$ is equi-integrable and $\left|\nabla\left(w_{j}-u_{j}\right)\right| \rightarrow 0$ in measure. By Proposition $3.8 \nabla w_{j}$ generates the same Young measure as $\nabla u_{j}$. Without loss of generality we can also assume that $w_{j} \rightarrow u_{0}$ in $W_{l o c}^{1, p}\left(\Omega ; \mathbf{R}^{m}\right)$.

Let $\Omega_{k} \subset \subset \Omega$ be an increasing sequence of sets with smooth boundary such that meas $\left(\Omega \backslash \Omega_{k}\right) \rightarrow 0$ as $k \rightarrow \infty$. Let $\phi_{k} \in C_{0}^{\infty}\left(\Omega_{k+1} ; \mathbf{R}^{m}\right)$ be a sequence such that $0 \leq \phi_{k} \leq 1, \phi=1$ on $\Omega_{k}$. Consider a sequence $v_{k}=u_{0}+\left(w_{j(k)}-u_{0}\right) \phi_{k}$. We will prove that there exists a subsequence $j(k) \rightarrow \infty$ such that the sequence $\left|\nabla v_{k}\right|^{p}$ is equi-integrable and $v_{k} \rightarrow u_{0}$ 
in $W^{1, p}\left(\Omega ; \mathbf{R}^{m}\right)$. We have

$$
\nabla v_{k}=\nabla u_{0}+\left(\nabla w_{j(k)}-\nabla u_{0}\right) \phi_{k}+\left(w_{j(k)}-u_{0}\right) \otimes \nabla \phi_{k} .
$$

Then

$$
\begin{gathered}
\left\|\nabla\left(v_{k}-w_{j(k)}\right)\right\|_{L^{p}} \leq\left\|\nabla w_{j(k)}\right\|_{L^{p}\left(\Omega \backslash \Omega_{k}\right)}+\left\|\nabla u_{0}\right\|_{L^{p}\left(\Omega \backslash \Omega_{k}\right)} \\
+\left\|w_{i(k)}-u_{0}\right\|_{L^{p}\left(\Omega_{k+1} \backslash \Omega_{k}\right)}\left\|\nabla \phi_{k}\right\|_{C}, \\
\left\|v_{k}-u_{0}\right\|_{L^{p}} \leq\left\|w_{j(k)}-u_{0}\right\|_{L^{p}\left(\Omega_{k+1}\right)} .
\end{gathered}
$$

First two terms in the right-hand side of the first inequality converge to zero for any choice of $j(k) \rightarrow \infty$, both the last term and the righthand side of the second inequality converge to zero for a special choice of $j(k) \rightarrow \infty$ since $w_{j}-u_{0} \rightarrow 0$ in $L_{l o c}^{p}(\Omega)$ as $j \rightarrow \infty$. Thus $\left|\nabla v_{k}\right|^{p}$ is equi-integrable for this choice of $j(k)$, and $v_{k} \rightarrow u_{0}$ in $W_{0}^{1, p}\left(\Omega ; \mathbf{R}^{m}\right)$. Because $\nabla v_{k}-\nabla w_{j(k)} \rightarrow 0$ in measure the sequence $\nabla v_{k}$ generates the same Young measure $\left(\nu_{x}\right)_{x \in \Omega}$ (cf. Proposition 3.8).

In order to meet the last requirement $v_{k} \in u_{0}+C_{0}^{\infty}\left(\Omega ; R^{m}\right)$ we can take the mollifiers with sufficiently small radii of the already obtained sequence $v_{k}$.

The second part of the proposition may be proved by the same arguments, taking $w_{j}=u_{j}$.

Proposition 4.4 is proved. QED

Proposition 4.5. - Let $\left(\nu_{x}^{j}\right)_{x \in \Omega}$ be a sequence of gradient p-Young measures such that $\left(\nu_{x}^{j}\right)_{x \in \Omega} \longrightarrow^{*}\left(\nu_{x}\right)_{x \in \Omega}$ as $j \rightarrow \infty$ and the underlying deformations $u^{j}$ are equi-bounded in $W^{1, p}\left(\Omega ; \mathbf{R}^{m}\right), p \in[1, \infty[$.

1) If $\int_{\Omega} \int_{\mathbf{R}^{n m}}\left(1+|v|^{p}\right) d \nu_{x}^{j} d x<c$ and $p>1$ then $\left(\nu_{x}\right)_{x \in \Omega}$ is a gradient p-Young measure.

2) If the sequence $\left(\nu_{x}^{j}\right)_{x \in \Omega}$ satisfies the tightness condition with the integrand $\left(1+|\cdot|^{p}\right)$ then $\left(\nu_{x}\right)_{x \in \Omega}$ is a gradient $p$-Young measure.

Proof. - By Proposition 4.4 for any fixed $j$ there exists a sequence $u_{k}^{j} \in u_{j}+C_{0}^{\infty}\left(\Omega ; \mathbf{R}^{m}\right)\left(u_{j}\right.$ is the underlying deformation for $\left.\left(\nu_{x}^{j}\right)_{x \in \Omega}\right)$ such that $\left(\delta_{\nabla u_{k}^{j}(x)}\right)_{x \in \Omega} \rightarrow^{*}\left(\nu_{x}^{j}\right)_{x \in \Omega}$ in $L_{w}\left(\Omega ; K_{1}\right)$ and $u_{k}^{j}-u_{j} \rightarrow 0$ in $W_{0}^{1, p}\left(\Omega ; \mathbf{R}^{m}\right)$ as $k \rightarrow \infty$, and the functions $\left|\nabla u_{k}^{j}\right|^{p}$ are equi-integrable. In particular Theorem 3.7 shows that

$$
\lim _{k \rightarrow \infty} \int_{\Omega}\left(1+\left|\nabla u_{k}^{j}(x)\right|^{p}\right) d x=\int_{\Omega} \int_{\mathbf{R}^{n m}}\left(1+|v|^{p}\right) d \nu_{x}^{j} d x .
$$


Because of the convergence $\left(\nu_{x}^{j}\right)_{x \in \Omega}-^{*}\left(\nu_{x}\right)_{x \in \Omega}$ by usual diagonalization arguments we may isolate a sequence $u_{k(j)}^{j}$ bounded in $W^{1, p}\left(\Omega ; \mathbf{R}^{m}\right)$ such that $\left(\delta_{\nabla u_{k(j)}^{j}(x)}\right)_{x \in \Omega} \rightarrow^{*}\left(\nu_{x}\right)_{x \in \Omega}$ in $L_{w}\left(\Omega ; K_{1}\right)$ as $j \rightarrow \infty$ and

$$
\int_{\Omega}\left(1+\left|\nabla u_{k(j)}^{j}(x)\right|^{p}\right) d x-\int_{\Omega} \int_{\mathbf{R}^{n m}}\left(1+|v|^{p}\right) d \nu_{x}^{j} d x \rightarrow 0, j \rightarrow \infty .
$$

Thus, the first assertion follows from Proposition 4.4.

To prove the second assertion note that the tightness condition implies

$$
\int_{\mathbf{R}^{n m}}\left(1+|v|^{p}\right) d \nu_{(\cdot)}^{j} \rightarrow \int_{\mathbf{R}^{n m}}\left(1+|v|^{p}\right) d \nu_{(\cdot)} \text { in } L^{1}(\Omega), j \rightarrow \infty,
$$

see Theorem 3.7. Now we proceed as before, and because of the convergence

$$
\int_{\Omega}\left(1+\left|\nabla u_{k(j)}^{j}(x)\right|^{p}\right) d x \rightarrow \int_{\Omega} \int_{\mathbf{R}^{n+m}}\left(1+|v|^{p}\right) d \nu_{x} d x
$$

by the same thcorcm we obtain that the functions $\left|\nabla u_{k(j)}^{j}\right|^{p}$ are equiintegrable. By the second part of Proposition $4.4\left(\nu_{x}\right)_{x \in \Omega}$ is a gradient p-Young measure.

Both assertions of Proposition 4.5 are proved. QED

Proof of Theorem 4.2. - Without loss of generality we may assume that $0 \in \Omega$. Consider first the case $\left(\nu_{x}\right)_{x \in \Omega}=\left(\delta_{\nabla u_{0}(x)}\right)_{x \in \Omega}$. Recall that $u_{0} \in l_{A}+W_{0}^{1, p}\left(\Omega ; \mathbf{R}^{m}\right)$.

For each $i \in \mathbf{N}$ consider a cover of $\Omega$ by disjoint sets $\Omega_{j}^{i}$ of the form $a_{j}^{i}+\epsilon_{j}^{i} \Omega(j \in \mathbf{N})$ with $\operatorname{diam} \Omega_{j}^{i} \leq 1 / i$, and a set $N_{i}$ of zero measure. Suppose also that for each $i^{\prime} \geq i, j^{\prime} \in \mathbf{N}$ either $\Omega_{j^{\prime}}^{i^{\prime}} \subset \Omega_{j}^{i}$ or $\Omega_{j^{\prime}}^{i^{\prime}} \cap \Omega_{j}^{i}=\emptyset$.

Define $v^{i}(x)=\epsilon_{j}^{i} u_{0}\left(\frac{x-a_{j}^{i}}{\epsilon_{j}^{i}}\right)$ for $x \in \Omega_{j}^{i}, v^{i}(x)=l_{A}(x)$ otherwise. Then

$$
\operatorname{Av}\left(\delta_{\nabla v^{i}(x)}\right)_{x \in \Omega_{j}^{i}}=\operatorname{Av}\left(\delta_{\nabla u_{0}(x)}\right)_{x \in \Omega} \text { for each } j
$$

We claim that

$$
\left(\delta_{\nabla v^{k}(x)}\right)_{x \in \Omega} \rightarrow^{*} \operatorname{Av}\left(\delta_{\nabla u_{0}(x)}\right)_{x \in \Omega}
$$

Let $\left(\delta_{\nabla v^{k}(x)}\right)_{x \in \Omega}$ be a subsequence (not relabeled) which generates a Young measure $\left(\nu_{x}\right)_{x \in \Omega}$. Let $\tilde{\Omega}:=\cap_{i}\left(\cup_{j} \tilde{\Omega}_{j}^{i}\right)$, where $\tilde{\Omega}_{j}^{i}$ is the set of interior points of $\Omega_{j}^{i}$. It is clear that meas $(\Omega \backslash \tilde{\Omega})=0$. For each $x_{0} \in \tilde{\Omega}$ there exists a Vol. $16, n^{\circ}$ 6-1999 
sequence $\Omega_{j(i)}^{i}$ such that $x_{0} \in \Omega_{j(i)}^{i}$ for each $i \in \mathbf{N}$. By the Lusin property (Theorem 3.2) and Lemma 3.4

$$
\operatorname{Av}\left(\nu_{x}\right)_{x \in \Omega_{j(i)}^{i}}-^{*} \nu_{x_{0}} \text { in } C_{0}\left(\mathbf{R}^{n m}\right)^{\prime} \text { as } i \rightarrow \infty
$$

for a.a. $x_{0} \in \tilde{\Omega}$. Because

$$
\begin{gathered}
\operatorname{Av}\left(\delta_{\nabla v^{k}(x)}\right)_{x \in \Omega_{j(i)}^{i}} \rightarrow^{*} \operatorname{Av}\left(\nu_{x}\right)_{x \in \Omega_{j(i)}^{i}} \text { in } C_{0}\left(\mathbf{R}^{n m}\right)^{\prime} \text { as } k \rightarrow \infty \text { and } \\
\operatorname{Av}\left(\delta_{\nabla v^{k}(x)}\right)_{x \in \Omega_{j(i)}^{i}}=\operatorname{Av}\left(\delta_{\nabla u_{0}(x)}\right)_{x \in \Omega} \text { for any } k \geq i
\end{gathered}
$$

we infer that $\operatorname{Av}\left(\nu_{x}\right)_{x \in \Omega_{j(i)}^{i}}=\operatorname{Av}\left(\delta_{\nabla u_{0}(x)}\right)_{x \in \Omega}$ for all $i \in \mathbf{N}$. Then $\nu_{x_{0}}=\operatorname{Av}\left(\delta_{\nabla u_{0}(x)}\right)_{x \in \Omega}$ for a.a. $x_{0} \in \tilde{\Omega}$.

Because each subsequence of the original sequence $\left(\delta_{\nabla v^{i}(x)}\right)_{x \in \Omega}$ contains a subsequence converging weakly* in $L_{u}\left(\Omega ; K_{1}\right)$ to the homogeneous Young measure $\operatorname{Av}\left(\delta_{\nabla u_{0}(x)}\right)_{x \in \Omega}$ we obtain that the original sequence has this property.

By Lemma 2.2 the functions $\left|\nabla v^{i}\right|^{p}$ are equi-integrable. Hence $\operatorname{Av}\left(\delta_{\nabla u_{0}(x)}\right)_{x \in \Omega} \in G M_{p}(A)$. In the case $u_{0} \in l_{A}+W_{0}^{1, \infty}\left(\Omega ; \mathbf{R}^{m}\right)$ we have that $v^{i}-l_{A} \rightarrow^{*} 0$ in $W_{0}^{1, \infty}\left(\Omega ; \mathbf{R}^{m}\right)$. Thus $\operatorname{Av}\left(\delta_{\nabla u_{0}(x)}\right)_{x \in \Omega} \in G M_{\infty}(A)$.

If $\left(\nu_{x}\right)_{x \in \Omega}$ is a gradient $\mathrm{p}$-Young measure with the underlying deformation $u_{0} \in l_{A}+W_{0}^{1, p}\left(\Omega ; \mathbf{R}^{m}\right)$ then by Proposition 4.4 there exists a sequence $u_{i} \in l_{A}+C_{0}^{\infty}\left(\Omega ; \mathbf{R}^{m}\right)$ generating $\left(\nu_{x}\right)_{x \in \Omega}$ as a gradient $\mathrm{p}$-Young measure (in this case the functions $\left|\nabla u_{i}\right|^{p}$ are equi-integrable). Because $\operatorname{Av}\left(\delta_{\nabla u_{i}(x)}\right)_{x \in \Omega} \longrightarrow^{*} \operatorname{Av}\left(\nu_{x}\right)_{x \in \Omega}$ and the modulus of equi-integrability of p-powers of gradients of sequences generating $\operatorname{Av}\left(\delta_{\nabla u_{i}(x)}\right)_{x \in \Omega}$ does not exceed modulus of equi-integrability of $\left|\nabla u_{i}\right|^{p}$ (cf. Lemma 2.2) we obtain that the sequence $\operatorname{Av}\left(\delta_{\nabla u_{i}(x)}\right)_{x \in \Omega}$ satisfies the requirements of the second assertion of Proposition 4.5. IIence $\operatorname{Av}\left(\nu_{x}\right)_{x \in \Omega} \in G M_{p}(A)$. This completes the proof of the first claim of the theorem.

Let us prove the second claim. Let $\left(\nu_{x}\right)_{x \in \Omega}$ be a gradient p-Young measure. There exists a sequence $\Omega_{k}$ of compact subsets of $\Omega$ such that $\operatorname{meas}\left(\Omega \backslash \Omega_{k}\right) \rightarrow 0$ as $k \rightarrow \infty$, the restrictions of $\nu_{(\cdot)}$ to $\Omega_{k}$ are continuous in $\rho$ metric, and all points of $\Omega_{k}$ are Lebesgue for the map $x \rightarrow\left\langle 1+|v|^{p} ; \nu_{x}\right\rangle$.

Let $\nabla u_{i}$ be a sequence generating $\left(\nu_{x}\right)_{x \in \Omega}$ as a gradient $p$-Young measure. Let $x_{0}$ be a Lebesgue point of $\Omega_{k}$ and let $B\left(x_{0}, \epsilon\right)\ulcorner\Omega$ for an $\epsilon>0$. For $j \in \mathbf{N}$ consider a sequence $u_{i}^{j}, i \in \mathbf{N}$, defined on $B\left(x_{0}, \epsilon\right) \subset \Omega$ by the formula $u_{i}^{j}\left(x_{0}+y\right)=j\left\{u_{i}\left(x_{0}+y / j\right)-u_{i}\left(x_{0}\right)\right\}$.

For $|y| \leq \epsilon$ we have that $\nabla u_{i}^{j}\left(x_{0}+y\right)=\nabla u_{i}\left(x_{0}+y / j\right)$. Then $\nabla u_{i}^{j}, i \in \mathbf{N}$, generate a gradient p-Young measure $\left(\nu_{x}^{j}\right)_{x \in B\left(x_{0}, \epsilon\right)}$ with $\nu_{x_{0}+y}^{j}=\nu_{x_{0}+y / j}$, 
$y \in B\left(x_{0}, \epsilon\right)$. By continuity of the restriction of $\nu: \Omega \rightarrow\left(K_{1}, \rho\right)$ to $\Omega_{k}$ and Proposition 3.4 we infer $\left(\nu_{x}^{j}\right)_{x \in B\left(x_{0}, \epsilon\right)} \rightarrow^{*} \nu_{x_{0}}$ in $L_{w}\left(B\left(x_{0}, \epsilon\right) ; K_{1}\right)$ (here $\nu_{x_{0}}$ is a homogeneous Young measure). Moreover, since $x_{0}$ is a Lebesgue point for the map $x \rightarrow\left\langle 1+|v|^{p} ; \nu_{x}\right\rangle$ we have

$$
\int_{B\left(x_{0}, \epsilon\right)}\left\langle 1+|v|^{p} ; \nu_{x}^{j}\right\rangle d x \rightarrow \int_{B\left(x_{0}, \epsilon\right)}\left\langle 1+|v|^{p} ; \nu_{x_{0}}\right\rangle d x, j \rightarrow \infty .
$$

Thus $\left(\nu_{x}^{j}\right)_{x \in B\left(x_{0}, \epsilon\right)}$ satisfies the tightness condition with the integrand $1+|\cdot|^{p}$ (cf. Theorem 3.7). By the second assertion of Proposition 4.5 we infer that $\nu_{x_{0}}$ is a homogeneous gradient $\mathrm{p}$-Young measure. This proves the second claim of the theorem.

The proof of the theorem is completed. QED

Proof of Corollary of Theorem 4.2. - It is obvious that $\inf _{\nu \in G M_{p}(A)}\langle L ; \nu\rangle \leq \inf _{\nu \in G M_{\infty}(A)}\langle L ; \nu\rangle$. The inequality

$$
\inf _{\nu \in G M_{\infty}(A)}\langle L ; \nu\rangle \leq \inf _{\phi \in C_{0}^{\infty}\left(\Omega ; \mathbf{R}^{m}\right)} \frac{1}{\operatorname{meas} \Omega} \int L(A+\nabla \phi(x)) d x
$$

holds because $\operatorname{Av}\left(\delta_{\nabla \phi(x)}\right)_{x \in \Omega} \in G M_{\infty}(A)$ (cf. Theorem 4.2). To prove the converse inequality

$$
\inf _{\phi \in C_{0}^{\infty}\left(\Omega ; \mathbf{R}^{m}\right)} \frac{1}{\text { meas } \Omega} \int L(A+\nabla \phi(x)) d x \leq \inf _{\nu \in G M_{p}(A)}\langle L ; \nu\rangle
$$

notice that by Proposition 4.4 a $\nu \in G M_{p}(A)$ is generated as a gradient p-Young measure by gradients of a sequence $u_{k} \in l_{A}+C_{0}^{\infty}\left(\Omega ; \mathbf{R}^{m}\right)$. In particular, by Theorem 3.7

$$
\int_{\Omega} L\left(\nabla u_{k}(x)\right) d x \rightarrow\left\langle L_{i} ; \nu\right\rangle \text { meas } \Omega \text { as } k \rightarrow \infty .
$$

The first assertion is proved. Let us prove the second one.

If $L$ is quasiconvex at $A$ then for any $k \in \mathbf{N}$ the inequality

$$
\int_{\Omega} L\left(\nabla u_{k}(x)\right) d x \geq L(A) \text { meas } \Omega
$$

holds with the above $u_{k}$, and, as a consequence, $\langle L ; \nu\rangle \geq L(A)$.

Conversely, if $\phi \in l_{A}+C_{0}^{\infty}\left(\Omega ; \mathbf{R}^{m}\right)$ then the inequality $\left\langle L ; \operatorname{Av}\left(\delta_{\nabla \phi(x)}\right)_{x \in \Omega}\right\rangle \geq L(A)$ holds because $\operatorname{Av}\left(\delta_{\nabla \phi(x)}\right)_{x \in \Omega} \in G M_{\infty}(A)$. This implies the inequality $\int_{\Omega} L(A+\nabla \phi(x)) d x \geq L(A)$ meas $\Omega$. Thus $L$ is quasiconvex at $A$.

Vol. $16, n^{\circ}$ 6-1999. 
The proof of the corollary is completed. QED

The proof of Theorem 4.3 from [29] is based on an abstract version of the Hahn-Banach theorem for special functional spaces, the relaxation theorem in the simplest form (Theorem 4.6), and some technical approximation results (see Lemma 5.1 and the proof of Theorem 1.1 from [29]). We also prove this result first in the homogeneous case through the relaxation theorem (we give a direct and self-contained proof of the latter theorem). In this case we propose a proof based on the integral representation of the metric $\rho$ and Proposition 4.5. This proof admits far-reaching extensions, see [47]. Then we extend the result to the general (nonhomogeneous) case utilizing construction in the proof of Theorem 3 from [44].

THEOREM 4.6. - Let $L: \mathbf{R}^{n m} \rightarrow \mathbf{R}$ be a continuous function satisfying the estimates

$$
A_{1}|v|^{p}+B_{1} \leq L(v) \leq A_{2}|v|^{p}+B_{2}, \quad p \in\left[1, \infty\left[, \quad A_{2} \geq A_{1}>0 .\right.\right.
$$

Then there exists a function $L^{q c}$, which is the greatest among all quasiconvex functions minorizing $L$. This function is given by the formula

$$
L^{q c}(A)=\inf _{\nu \in G M_{p}(A)} \int_{\mathbf{R}^{n m}} L(v) d \nu,
$$

where $G M_{p}(A)$ is the set of all gradient p-Young measures with the center of mass at $A$. Moreover $L^{q c}$ is continuous and satisfies the same estimates as $L$.

There are proofs of this theorem not involving Young measures (see [14], [15]). In this case $L^{q c}(A)$ is defined first as

$$
\inf _{\phi \in C_{0}^{\infty}\left(\Omega ; \mathbf{R}^{m}\right)} \frac{1}{\operatorname{meas} \Omega} \int_{\Omega} L(A+\nabla \phi(x)) d x .
$$

Then the result follows from the corollary of Theorem 4.2. We propose here a proof which can easily be extended to the case of dependence of $L$ on the lower order terms (see the proof of Theorem 1.2 in $\$ 5$ ), but involves Theorem 2.1.

Proof. - Consider first the case $p>1$.

By Theorem 3.7 and Proposition 4.5 the infimum of $I(\nu):=$ $\int_{\mathbf{R}^{n m}} L(v) d \nu$ over $G M_{p}(A)$ is attained. Let $V(A)$ denote the set of minimizers of this problem. 
If $A_{k} \rightarrow A$ as $k \rightarrow \infty$ and $\nu_{k} \in V\left(A_{k}\right)$ then for a subsequence $\nu_{j}$ we have

$$
\lim _{j \rightarrow \infty} I\left(\nu_{j}\right)=\liminf _{k \rightarrow \infty} I\left(\nu_{k}\right), \nu_{j} \rightarrow^{*} \nu
$$

where $\nu$ is automatically a gradient p-Young measure with the center of mass at $A$ (cf. Proposition 4.5). By Theorem $3.7 \lim _{j \rightarrow \infty} I\left(\nu_{j}\right) \geq I(\nu)$. Thus, $\liminf _{k \rightarrow \infty} L^{q c}\left(A_{k}\right) \geq L^{q c}(A)$, i.e. $L^{q c}$ is lower semicontinuous at $A$.

To prove upper semicontinuity notice that if $\nu \in V(A)$ then the measures $\nu \diamond A_{k}$ are gradient p-Young measures centered at $A_{k}$, respectively, and

$$
\nu_{k} \rightarrow^{*} \nu, \limsup _{k \rightarrow \infty} L^{q c}\left(A_{k}\right) \leq \lim _{k \rightarrow \infty} I\left(\nu_{k}\right)=I(\nu) .
$$

Therefore $L^{q c}$ is continuous.

Since each function $\phi \in C_{0}^{\infty}\left(\Omega ; \mathbf{R}^{m}\right)$ can be approximated in $W^{1, \infty}$ norm by piecewise affine ones, to establish quasiconvexity it is enough to prove the inequality

$$
\int_{\Omega} L^{q c}(\Lambda \mid \nabla \phi(x)) d x \geq L^{q c}(\Lambda) \operatorname{mcas} \Omega
$$

for piecewise affine functions $\phi \in W_{0}^{1, \infty}\left(\Omega ; \mathbf{R}^{m}\right)$. Fix such a $\phi$. Let $\Omega_{j}(j=1, \ldots, k)$ be a finite collection of subdomains of $\Omega$ on which $A+\nabla \phi$ has constant values $A_{1}, \ldots, A_{k}$ respectively. Let $\nu_{j} \in V\left(A_{j}\right)$, $j \in\{1, \ldots, k\}$.

By Proposition 4.4 there exist functions $u_{j} \in C_{0}^{\infty}\left(\Omega_{j} ; \mathbf{R}^{m}\right)$ such that

$$
\int_{\Omega_{j}} L^{q c}\left(A_{j}\right) d x=\int_{\Omega_{j}} \int_{\mathbf{R}^{n m}} L(v) d \nu_{j} d x \geq \int_{\Omega_{j}} L\left(A_{j}+\nabla u_{j}(x)\right) d x-\epsilon / k .
$$

Define $\tilde{u}(x)$ as $\phi(x)+u_{j}(x)$ for $x \in \Omega_{j}, j \in\{1, \ldots, k\}$, and as $\phi(x)$ otherwise. We have

$$
\begin{gathered}
\int_{\Omega} L^{q c}(A+\nabla \phi(x)) d x=\int_{\Omega \backslash \cup_{j=1}^{k} \Omega_{j}} L^{q c}(A+\nabla \phi(x)) d x+\sum_{j=1}^{k} \int_{\Omega_{j}} L^{q c}\left(A_{j}\right) d x \geq \\
\int_{\Omega \backslash \cup_{j=1}^{k} \Omega_{j}} L^{q c}(A+\nabla \phi(x)) d x+\sum_{j=1}^{k} \int_{\Omega_{j}} L\left(A_{j}+\nabla u_{j}(x)\right) d x-\epsilon \geq \\
\int_{\Omega} L(A+\nabla \tilde{u}(x)) d x-\epsilon+\int_{\Omega \backslash \cup_{j=1}^{k} \Omega_{j}}\left\{L^{q c}(A+\nabla \phi(x))-L(A+\nabla \phi(x))\right\} d x .
\end{gathered}
$$


The first term in the right-hand side of the inequality exceeds $L^{q c}(A)$ meas $\Omega$ since

$$
\int_{\Omega} L(A+\nabla \tilde{u}(x)) d x=\left\langle L ; \operatorname{Av}\left(\delta_{\nabla \tilde{u}(x)}\right)_{x \in \Omega}\right\rangle \text { meas } \Omega,
$$

where $\operatorname{Av}\left(\delta_{\nabla \tilde{u}(x)}\right)_{x \in \Omega} \in G M_{p}(A)$ by Theorem 4.2. The second term tends to zero as $k \rightarrow \infty$. Because $\epsilon>0$ may be chosen arbitrary small we obtain that

$$
\int_{\Omega} L^{q c}(A+\nabla \phi(x)) d x \geq L^{q c}(A) \operatorname{meas} \Omega .
$$

This proves quasiconvexity of $L^{q c}$.

By the construction $L^{q c}$ is the greatest function among quasiconvex ones minorizing $L$. Indeed, if $F$ is a quasiconvex function minorizing $L$ then for any $\nu \in V(A)$ we have

$$
L^{q c}(A)=\langle L ; \nu\rangle \geq\langle F ; \nu\rangle \geq F(A)
$$

where the last inequality follows from the corollary of Theorem 4.2. Hence $L^{q c}(v) \geq A_{1}+B_{1}|v|^{p}$, while the estimate $A_{2}+B_{2}|v|^{p} \geq L(v) \geq L^{q c}(v)$ is obvious.

The theorem is proved in the case $p>1$.

Consider the remaining case $p=1$. To treat this case consider a family of auxiliary integrands $L_{\mu}(\cdot):=L(\cdot)+\mu|\cdot|^{2}, \mu>0$, and their quasiconvexifications $L_{\mu}^{q c}$. For any fixed $v \in \mathbf{R}^{n m}$ the values $I_{\mu}^{q c}(v)$ decrease to $\tilde{L}(v)$ as $\mu \rightarrow 0$. Because $L_{\mu}^{q c}$ are continuous functions bounded below by $B_{1}$ the function $\tilde{L}$ is upper semicontinuous and is bounded below by $B_{1}$.

Because the inequality

$$
\int_{\Omega} L_{\mu}^{q c}(A+\nabla \phi(x)) d x \geq L_{\mu}^{q c}(A) \text { meas } \Omega
$$

holds for any $\phi \in C_{0}^{\infty}\left(\Omega ; \mathbf{R}^{m}\right)$ and $A \in \mathbf{R}^{n m}$ the same holds for the integrand $\tilde{L}$ by monotone convergence theorem and then $\tilde{L}$ is quasiconvex.

To establish continuity of $L$ it is enough to prove lower semicontinuity. The latter follows from quasiconvexity of $\tilde{L}$. In fact if $A_{k} \rightarrow A$ then there cxist functions $\phi_{k} \in l_{A}+C_{0}^{\infty}\left(\Omega ; \mathbf{R}^{m}\right)$ such that $\operatorname{mcas}\left\{x \in \Omega: \phi_{k}(x) \neq\right.$ $\left.A_{k}\right\} \rightarrow 0$ and $\left\|\phi_{k}\right\|_{W^{1, \infty}} \leq c<\infty$. Then

$$
\tilde{L}\left(A_{k}\right) \operatorname{meas} \Omega-\int_{\Omega} \tilde{L}\left(A+\nabla \phi_{k}(x)\right) d x \rightarrow 0 .
$$


Since the second term exceeds $\tilde{L}(A)$ meas $\Omega$ we infer that $\liminf _{k \rightarrow \infty} \tilde{L}\left(A_{k}\right) \geq \tilde{L}(A)$.

Therefore $\tilde{L}(A)$ is a continuous quasiconvex function. By the Dini convergence theorem $L_{\mu}^{q c} \rightarrow \tilde{L}$ as $\mu$, +0 locally uniformly. Because $L_{\mu}^{q c}$ are the greatest functions among quasiconvex ones minorizing the integrands $L(\cdot)+\mu|\cdot|^{2}$ the function $\tilde{L}$ is the greatest among quasiconvex ones minorizing the original integrand $L$.

In order to prove

$$
\tilde{L}(A)=\inf _{\nu \in G M_{1}(A)}\langle L ; \nu\rangle
$$

notice that by Corollary of Theorem 4.2 the following holds

$$
\begin{gathered}
\tilde{L}(A)=\lim _{\mu \rightarrow+0} L_{\mu}^{q c}(A)=\lim _{\mu \rightarrow+0} \inf _{\nu \in G M_{2}(A)}\left\langle L(\cdot)+\mu|\cdot|^{2} ; \nu\right\rangle= \\
\lim _{\mu \rightarrow+0} \inf _{\nu \in G M_{\infty}(A)}\left\langle L(\cdot)+\mu|\cdot|^{2} ; \nu\right\rangle=\inf _{\nu \in G M_{\infty}(A)}\langle L ; \nu\rangle=\inf _{\nu \in G M_{1}(A)}\langle L ; \nu\rangle .
\end{gathered}
$$

This completes the proof of Theorem 4.6. QED

Proof of Theorem 4.3. - Necessity of the conditions (i)-(iii) follows from Theorem 3.7, Theorem 4.2 and its corollary. To prove their sufficiency we will prove first the theorem in the homogeneous case. In this case the result follows from the integral representation for the metric $\rho$. The result will be then extended to the general case following the construction in the proof of Theorem 3 from [44].

In the homogeneous case $\left(\nu_{x}\right)_{x \in \Omega}$ does not depend on $x$ (we will denote this measure as $\nu$ ). Recall that $G M_{p}(A)$ denotes the set of all homogeneous gradient p-Young measures with the center of mass at $A$.

We prove first that $G M_{p}(A)$ is a convex set. Let $\nu^{1}, \nu^{2} \in G M_{p}(A)$, $\lambda \in] 0,1\left[\right.$. Let $\Omega_{1}, \Omega_{2}$ be disjoint open subsets of $\Omega$ such that meas $\left(\partial \Omega_{1}\right)=$ $\operatorname{meas}\left(\partial \Omega_{2}\right)=0$ and $\operatorname{meas} \Omega_{1}=\lambda$ meas $\Omega, \operatorname{meas} \Omega_{2}=(1-\lambda)$ meas $\Omega$. By Proposition 4.4 there exist sequences $u_{i}^{1} \in l_{A}+C_{0}^{\infty}\left(\Omega_{1} ; \mathbf{R}^{m}\right)$, $u_{i}^{2} \in l_{A}+C_{0}^{\infty}\left(\Omega_{2} ; \mathbf{R}^{m}\right)$ generating $\nu^{1}$ and $\nu^{2}$ as gradient $\mathrm{p}$-Young measures respectively. Hence the measure, which equals $\nu^{1}$ on $\Omega_{1}, \nu^{2}$ on $\Omega_{2}$. is a gradient p-Young measure. By Theorem 4.2 its average, which is $\lambda \nu^{1}+(1-\lambda) \nu^{2}$, is also a gradient $\mathrm{p}$-Young measure. This proves convexity of $G M_{p}(A)$.

Let $\Phi_{0}=\left(1+|\cdot|^{p}\right)$. To prove the inclusion $\nu \in G M_{p}(A)$ it is enough to prove existence of a sequence $\nu_{k} \in G M_{p}(A)$ such that

$$
\rho\left(\nu_{k}, \nu\right)+\left|\left\langle\Phi_{0} ; \nu_{k}\right\rangle-\left\langle\Phi_{0} ; \nu\right\rangle\right| \rightarrow 0, k \rightarrow \infty .
$$

Vol. $16, n^{\circ}$ 6-1999. 
In fact, the convergence of the first term to zero means that $\nu_{k}$ generates $\nu$. Then, by Proposition 4.5 convergence of the second term to zero implies $\nu \in G M_{p}(A)$.

We will prove $(4.1)$ by contradiction. Recall that

$$
\rho\left(\nu_{k}, \nu\right):=\sum_{i=1}^{\infty} \frac{1}{2^{i}\left\|\Phi_{i}\right\|_{C}}\left|\left\langle\Phi_{i} ; \nu_{k}\right\rangle-\left\langle\Phi_{i} ; \nu\right\rangle\right|,
$$

where the sequence $\left\{\Phi_{i}\right\}$ is dense in $C_{0}\left(\mathbf{R}^{n m}\right)$.

If (4.1) does not hold, then for a sufficiently large $l$ and an $\epsilon>0$ we have

$$
\inf _{\mu \in G M_{p}(A)}\left\{\left|\left\langle\Phi_{0} ; \mu\right\rangle-\left\langle\Phi_{0} ; \nu\right\rangle\right|+\sum_{i=1}^{l} \frac{1}{2^{i}\left\|\Phi_{i}\right\|_{C}}\left|\left\langle\Phi_{i} ; \mu\right\rangle-\left\langle\Phi_{i} ; \nu\right\rangle\right|\right\}>\epsilon .
$$

Then, the subset of $\mathbf{R}^{l+1}$ given by the vectors

$$
\left(\left\langle\Phi_{0} ; \mu\right\rangle, \frac{1}{2\left\|\Phi_{1}\right\|}\left\langle\Phi_{1} ; \mu\right\rangle, \ldots, \frac{1}{2^{l}\left\|\Phi_{l}\right\|}\left\langle\Phi_{l} ; \mu\right\rangle\right), \mu \in G M_{p}(A),
$$

is convex in view of convexity of $G M_{p}(A)$, and the vector generated by $\nu$ does not belong to its closure. Hence, there exists a vector $c \in \mathbf{R}^{l+1}$ such that

$$
\underset{\mu \in G M_{p}(A)}{\inf } \sum_{i=0}^{l} c_{i}\left\langle\Phi_{i} ; \mu\right\rangle>\sum_{i=0}^{l} c_{i}\left\langle\Phi_{i} ; \nu\right\rangle+\delta, \delta>0 .
$$

Then

$$
\inf _{\mu \in G M_{p}(A)}\langle L ; \mu\rangle>\langle L ; \nu\rangle+\delta, \text { with } L=\sum_{i=0}^{l} c_{i} \Phi_{i} .
$$

Note that the coefficient $c_{0}$ can not be negative - otherwise the value at the left-hand side is $-\infty$. In the case $c_{0}=0$ we can replace $L$ by $L+\eta \Phi_{0}$ and (4.3) still holds for $\eta>0$ sufficiently small. Note now that this integrand $L$ satisfies conditions of Theorem 4.6 and that the left-hand side in (4.3) is equal to $L^{q c}(A)$. Since $L \geq L^{q c}$ everywhere we infer that $L^{q c}(A)>\left\langle L^{q c} ; \nu\right\rangle+\delta$, that contradicts the assumption (ii) of the theorem.

The above contradiction proves that $\nu \in G M_{p}(A)$. Hence the theorem is proved in the homogeneous case.

Let $\nu$ be a homogeneous gradient p-Young measure with the center of mass at $A$ and let $\tilde{\Omega}$ be an open subset of $\Omega$ such that meas $(\partial \tilde{\Omega})=0$. The Young measure $\left(\nu \diamond \nabla u_{0}(x)\right)_{x \in \tilde{\Omega}}$ is also a gradient $\mathrm{p}$-Young measure. 
Indeed, if $\nu$ is generated by $w_{i} \in l_{A}+C_{0}^{\infty}\left(\tilde{\Omega} ; \mathbf{R}^{m}\right)$ as a gradient p-Young measure then $\left(\nu \diamond \nabla u_{0}(x)\right)_{x \in \tilde{\Omega}}$ is generated by gradients of the sequence $w_{i}-l_{A}+u_{0}$. Because $w_{i}-l_{A} \in C_{0}^{\infty}\left(\tilde{\Omega} ; \mathbf{R}^{m}\right)$ the measure, which equals $\nu \diamond \nabla u_{0}(x)$ for $x \in \tilde{\Omega}$ and $\delta_{\nabla u_{0}(x)}$ for other $x \in \Omega$, is gradient $\mathrm{p}$-Young one.

By Proposition 2.3 we can prove also that if $\Omega_{1}, \ldots, \Omega_{l}$ are disjoint measurable subsets of $\Omega, \nu_{1}, \ldots, \nu_{l}$ are homogeneous p-Young measures with the centers of mass at $A_{1}, \ldots, A_{l}$ respectively then the measure $\left(\tilde{\nu}_{x}\right)_{x \in \Omega}$, which equals $\nu_{i} \diamond \nabla u_{0}(x)$ for $x \in \Omega_{i}(i=1, \ldots, l), \delta_{\nabla u_{0}(x)}$ for other $x \in\left(\Omega \backslash \cup_{i=1}^{l} \Omega_{i}\right)$, is also a gradient $\mathrm{p}$-Young measure. To prove this notice first that if $\Omega_{i}$ are open sets with meas $\left(\partial \Omega_{i}\right)=0$ then the claim follows from the result of the previous paragraph. If $\Omega_{i}$ are compact sets then the claim follows from Proposition 2.3 and Lemma 2.2. In the general case the same arguments let us prove the claim by approximating $\Omega_{i}$ with compact subsets.

Consider now the general case of nonhomogeneous measure $\left(\nu_{x}\right)_{x \in \Omega}$. For each $k \in \mathbf{N}$ there exists a compact subset $\Omega_{k}$ of $\Omega$ such that $\operatorname{meas}\left(\Omega \backslash \Omega_{k}\right) \leq 1 / k$, the restrictions of $u_{0}, \nabla u_{0}$ and $\int_{\mathbf{R}^{n m}}\left(1+|v|^{p}\right) d \nu_{(\cdot)}$ to $\Omega_{k}$ are continuous, the restriction of $\nu: \Omega \rightarrow\left(K_{1}, \rho\right)$ to $\Omega_{k}$ is continuous, and for each $x \in \Omega_{k}$ the measure $\nu_{x}$ satisfies the condition (ii) of the theorem.

We will prove that the measure $\left(\nu_{x}^{k}\right)_{x \in \Omega}$ defined as $\nu_{x}$ for $x \in \Omega_{k}$, and as $\delta_{\nabla u_{0}(x)}$ for $x \in\left(\Omega \backslash \Omega_{k}\right)$, is a gradient p-Young measure. By the second part of Proposition 4.5 this result will be enough to complete the proof of the theorem. Indeed, in this case $\left(\nu_{x}^{k}\right)_{x \in \Omega} \rightarrow^{*}\left(\nu_{x}\right)_{x \in \Omega}$ in $L_{w}\left(\Omega ; K_{1}\right)$ and $\left(\nu_{x}^{k}\right)_{x \in \Omega}$ satisfies conditions of the second assertion of Proposition 4.5.

Fix $k \in \mathbf{N}$. Suppose that $C=\left[-a, a\left[^{n}\right.\right.$ contains $\Omega_{k}$ and let $C_{j}^{1}$ $\left(j=1, \ldots, 2^{n}\right)$ be quadrants of $C$. For each $i>1$ decompose $C_{j}^{i}$ $\left(j=1, \ldots, 2^{n i}\right)$ in $2^{n}$ cubes of equal size in the similar way. Let $B_{j}^{i}=C_{j}^{i} \cap \Omega_{k}\left(i \in \mathbf{N} ; j \in\left\{1, \ldots, 2^{n i}\right\}\right)$. Fix $i \in \mathbf{N}$. Let $x_{j} \in B_{j}^{i}$, let $\nu_{x}^{i, k}$ be equal to $\nu\left(x_{j}\right) \diamond \nabla u_{0}(x)$ for $x \in B_{j}^{i}, j \in\left\{1, \ldots, 2^{n i}\right\}$, and to $\delta_{\nabla u_{0}(x)}$ otherwise. Then $\left(\nu_{x}^{i, k}\right)_{x \in \Omega}$ is a gradient p-Young measure by the claim proved above.

Because the restriction of $\nu^{k}: \Omega \rightarrow\left(K_{1}, \rho\right)$ to $\Omega_{k}$ is continuous in $\rho$ metric we obtain that $\left(\nu_{x}^{i, k}\right)_{x \in \Omega} \rightarrow^{*}\left(\nu_{x}^{k}\right)_{x \in \Omega}$ as $i \rightarrow \infty$ (cf. Lemma 3.4). Moreover, the scquence $\left(\nu_{x}^{i, k}\right)_{x \in \Omega}, i \in \mathbf{N}$, satisfies the requirements of the second claim of Proposition 4.5 - tightness with the integrand $1+|\cdot|^{p}$. To prove this notice that because of continuity of the restrictions of $\nabla u_{0}, \int_{\mathbf{R}^{n m}}\left(1+|v|^{p}\right) d \nu_{(.)}$to $\Omega_{k}$ the family of homogeneous measures $\mu_{x, y}:=\nu_{x} \diamond \nabla u_{0}(y)$, where $x, y \in \Omega_{k}$, satisfies the tightness requirement: 
$\int_{\mathbf{R}^{n m} \backslash B(0, M)}\left(1+|v|^{p}\right) d \ell_{x, y} \rightarrow 0$ as $M \rightarrow \infty$ uniformly with respect to $x, y \in \Omega_{k}$. Actually, the family $\left\{\nu_{x}: x \in \Omega_{k}\right\}$ satisfies this requirement in view of continuity of the restriction of $\int_{\mathbf{R}^{n m}}\left(1+|v|^{p}\right) d \nu_{(\cdot)}$ to $\Omega_{k}$ and Theorem 3.7. Because $\sup _{y \in \Sigma_{k}}\left|\nabla u_{0}(y)\right|<\infty$ we obtain that the whole family $\mu_{x, y}$ satisfies this requirement.

By the second assertion of Proposition $4.5\left(\nu_{x}^{k}\right)_{x \in \Omega}$ is a gradient p-Young measure for each $k \in \mathbf{N}$.

This completes the proof of the theorem. QED

\section{OPTIMAL RESULTS ON RELAX AND CONVERGENCE IN ENERGY}

In this section we prove Theorems 1.2 and 1.4 stated in the introduction. Before proving these theorems we will first prove a version of lower semicontinuity theorem from [1] for completeness. The proof follows arguments from [29], [30], [39].

THeOREM 5.1. - Let $L: \Omega \times \mathbf{R}^{m} \times \mathbf{R}^{n m} \rightarrow \mathbf{R}$ be a Caratheodory integrand such that $|L(x, u, v)| \leq A|v|^{p}+B, u_{0} \in W^{1, p}\left(\Omega ; \mathbf{R}^{m}\right), p \in[1, \infty \mid$.

I) If the function $L\left(x, u_{0}(x), \cdot\right)$ is quasiconvex at $\nabla u_{0}(x)$ for a.a. $x \in \Omega$ then $\liminf \operatorname{in}_{k \rightarrow \infty} I\left(u_{k}\right) \geq I\left(u_{0}\right)$ for any sequence $u_{k} \rightarrow u_{0}$ in $W^{1, p}\left(\Omega: \mathbf{R}^{m}\right)$ such that the negative parts of $L\left(x, u_{k}(x), \nabla u_{k}(x)\right)$ are equi-integrable.

2) Conversely, if $\liminf \operatorname{in}_{k \rightarrow \infty} I\left(u_{k}\right) \geq I\left(u_{0}\right)$ for any $u_{k}-u_{0}$ in $W^{1, p}\left(\Omega ; \mathbf{R}^{m}\right)$ such that the sequence $\left|\nabla u_{k}\right|^{p}$ is equi-integrable then for a.e. $x \in \Omega$ the function $L\left(x, u_{0}(x), \cdot\right)$ is quasiconvex at $\nabla u_{0}(x)$.

Proof. - Without loss of generality we may assume that $\nabla u_{k}$ generates a gradient p-Young measure $\left(\nu_{x}\right)_{x \in \Omega}$ and $\left(u_{k}, \nabla u_{k}\right)$ generates Young measure $\left(\delta_{u_{0}(x)} \otimes \nu_{x}\right)_{x \in \Omega}$. By Theorem 3.7

$$
\liminf _{k \rightarrow \infty} I\left(u_{k}\right) \geq \int_{\Omega} \int_{\mathbf{R}^{n ! m}} L\left(x, u_{0}(x), \cdot\right) d \nu_{x} d x .
$$

By Proposition 4.4 and the Localization principle (see Theorem 4.2) for almost all $x \in \Omega$ the measure $\nu_{x}$ is a homogeneous gradient $\mathrm{p}$-Young measure. In view of quasiconvexity at appropriate points we have

$$
\int_{\mathbf{R}^{n m}} L\left(x, u_{0}(x), \cdot\right) d \nu_{x} \geq L\left(x, u_{0}(x), \nabla u_{0}(x)\right)
$$

for a.e. $x \in \Omega$ (cf. Corollary to Theorem 4.2). This proves the first part of the theorem. 
We will prove the second one by contradiction. Let $\Omega_{k} \subset \Omega$ be an increasing sequence of compact sets such that meas $\left(\Omega \backslash \Omega_{k}\right) \leq 1 / k$, the restrictions of $u_{0}, \nabla u_{0}$ to $\Omega_{k}$ and the restrictions of $L$ to $\Omega_{k} \times \mathbf{R}^{m} \times \mathbf{R}^{n m}$ are continuous. Suppose that for a I ebesgue point $x_{0}$ of $\Omega_{k}$ the function $L\left(x_{0}, u_{0}\left(x_{0}\right), \cdot\right)$ is not quasiconvex at $\nabla u_{0}\left(x_{0}\right)$. By Corollary to Theorem 4.2 there exists a $\nu \in G M_{p}\left(\nabla u_{0}\left(x_{0}\right)\right)$ and $\epsilon>0$ such that

$$
L\left(x_{0}, u_{0}\left(x_{0}\right), \nabla u_{0}\left(x_{0}\right)\right)>\int_{\mathbf{R}^{n m}} L\left(x_{0}, u_{0}\left(x_{0}\right), \cdot\right) d \nu+\epsilon .
$$

The same is true for all $x \in \Omega_{k}$ sufficiently close to $x_{0}$ and $\nu_{x}$ obtained from $\nu$ by exchanging the center of mass from $\nabla u_{0}\left(x_{0}\right)$ to $\nabla u_{0}(x)$. A Young measure, which equals $\nu_{x}$ for such $x$ and $\delta_{\nabla u_{0}(x)}$ for other $x \in \Omega$, is a gradient p-Young measure due to Theorem 4.3. By the last inequality lower semicontinuity fails along a sequence associated with this Young measure.

The proof of the theorem is completed. QED

Proof of the Theorem 1.2. - Let $\Omega_{k} \subset \Omega$ be a sequence of compact sets such that meas $\left(\Omega \backslash \Omega_{k}\right) \rightarrow 0$ as $k \rightarrow \infty$, and the restrictions of $L$ to $\Omega_{k} \times \mathbf{R}^{m} \times \mathbf{R}^{n m}$ are continuous. By Theorem 4.6 for each $(x, u) \in \Omega_{k} \times \mathbf{R}^{m}$ the function

$$
L^{q c}(x, u, \cdot)=\inf _{\nu \in G M_{p}(\cdot)} \int L(x, u, v) d \nu(v)
$$

is continuous and quasiconvex. Moreover it satisfies the estimates

$$
B_{1}+A_{1}|v|^{p} \leq L^{q c}(x, u, v) \leq B_{2}+A_{2}|v|^{p}
$$

and is the greatest function among quasiconvex functions minorizing the original one.

Let $V(x, u, A)$ be the set of all solutions to the problem

$$
\int_{\nu \in G M_{p}(A)} L(x, u, \cdot) d \nu \rightarrow \min .
$$

By Theorem 3.7 and the first assertion of Proposition $4.5 V(x, u, A)$ is a nonempty compact set in the metric space $\left(K_{1}, \rho\right)$ (see $\S 3$ ). We will prove continuity of the restriction of $L^{q c}$ to $\Omega_{k} \times \mathbf{R}^{m} \times \mathbf{R}^{n m}$ by arguments from the proof of Theorem 4.6.

Let $\left(x_{h}, u_{h}, v_{h}\right) \rightarrow\left(x_{0}, u_{0}, v_{0}\right)$ as $h \rightarrow \infty, \nu_{h} \in V\left(x_{h}, u_{h}, v_{h}\right)$. Then

$$
\liminf _{h \rightarrow \infty}\left\langle L\left(x_{h}, u_{h}, \cdot\right) ; \nu_{h}\right\rangle=\liminf _{h \rightarrow \infty} L^{q c}\left(x_{h}, u_{h}, v_{h}\right) .
$$

Vol. $16, n^{\circ}$ 6-1999. 
Moreover, for a subsequence $\nu_{j}$ of $\nu_{h}$ we have $\liminf _{h \rightarrow \infty}\left\langle L\left(x_{h}, u_{h}, \cdot\right) ; \nu_{h}\right\rangle=\lim _{j \rightarrow \infty}\left\langle L\left(x_{j}, u_{j}, \cdot\right) ; \nu_{j}\right\rangle, \nu_{j} \rightarrow^{*} \nu_{0}$ in $C_{0}\left(\mathbf{R}^{n m}\right)^{\prime}$.

By the first assertion of Proposition $4.5 \nu_{0} \in G M_{p}\left(v_{0}\right)$. By Theorem 3.7

$$
\left\langle L\left(x_{0}, u_{0}, \cdot\right) ; \nu_{0}\right\rangle \leq \liminf _{h \rightarrow \infty}\left\langle L\left(x_{h}, u_{h}, \cdot\right) ; \nu_{h}\right\rangle .
$$

This proves lower semicontinuity of the restriction of $L^{q c}$ to $\Omega_{k} \times \mathbf{R}^{m} \times \mathbf{R}^{n m}$ at $\left(x_{0}, u_{0}, v_{0}\right)$.

In order to prove upper semicontinuity notice that if $\nu_{0} \in V\left(x_{0}, u_{0}, v_{0}\right)$ then $\nu_{0} \diamond v_{h} \in G M_{p}\left(v_{h}\right)$ and $\left\langle 1+|\cdot|^{p} ; \nu_{0} \diamond v_{h}\right\rangle \rightarrow\left\langle 1+|\cdot|{ }^{p} ; \nu_{0}\right\rangle$ as $h \rightarrow \infty$. By Theorem 3.7 this implies convergence

$$
\left\langle L\left(x_{h}, u_{h}, \cdot\right) ; \nu_{0} \diamond v_{h}\right\rangle \rightarrow\left\langle L\left(x_{0}, u_{0}, \cdot\right) ; \nu_{0}\right\rangle
$$

Therefore

$\limsup _{h \rightarrow \infty} L^{q c}\left(x_{h}, u_{h}, v_{h}\right) \leq \limsup _{h \rightarrow \infty}\left\langle L\left(x_{h}, u_{h}, \cdot\right) ; \nu_{0} \diamond v_{h}\right\rangle-\left\langle L\left(x_{0}, u_{0}, \cdot\right) ; \nu_{0}\right\rangle$.

This proves upper semicontinuity of the restriction of $L^{q c}$ at $\left(x_{0}, u_{0}, v_{0}\right)$. Thus, we have proved continuity of the restriction of $L^{q c}$ to $\Omega_{k} \times \mathbf{R}^{m} \times \mathbf{R}^{n m}$ for every $k$.

Fix $u \in W^{1, p}\left(\Omega ; \mathbf{R}^{m}\right)$ and consider compact sets $\tilde{\Omega}_{k} \subset \Omega_{k}$ such that $\operatorname{meas}\left(\Omega_{k} \backslash \tilde{\Omega}_{k}\right) \leq 1 / k$ and the restrictions of $u, \nabla u$ to $\tilde{\Omega}_{k}$ are continuous.

Consider the multivalued mapping $W: x \in \Omega \rightarrow V\left(x, u_{0}(x), \nabla u_{0}(x)\right)$. Let $x$ be such that the function $L\left(x, u_{0}(x), \cdot\right): \mathbf{R}^{n m} \rightarrow \mathbf{R}$ is continuous. Then $V\left(\nabla u_{0}(x)\right)$ is a nonempty compact set in the metric $\rho$ introduced in $\S 3$ in view of Theorem 3.7 and the first part of Proposition 4.5. Thus $W(x)$ is closed for a.e. $x \in \Omega$. Because of continuity of the restriction of $L^{q c}\left(\cdot, u_{0}(\cdot), \nabla u_{0}(\cdot)\right)$ to $\tilde{\Omega}_{k}$ the restriction of $W$ to $\tilde{\Omega}_{k}$ is upper semicontinuous: if $\nu_{k} \in W\left(x_{k}\right), x_{k} \rightarrow x$ and $\rho\left(\nu_{k}, \nu\right) \rightarrow 0$ (this is the same as $\nu_{k} \rightarrow^{*} \nu$ ) then $\nu \in W(x)$. Thus, $W$ is measurable in $\Omega$.

By Theorem 3.5 there exists a measurable selection of $W$. By theorems $3.3,4.3$ this selection is a gradient p-Young measure. For a sequence $u_{k}$ associated with $\left(\nu_{x}\right)_{x \in \Omega}$ we have that the sequence $\left(u_{k}, \nabla u_{k}\right)$ generates the Young measure $\left(\delta_{u_{0}(x)} \otimes \nu_{x}\right)_{x \in \Omega}$ and the functions $\left|\nabla u_{k}\right|^{p}$ are equiintegrable. Hence Theorem 3.7 yields

$$
\begin{aligned}
I\left(u_{k}\right) \rightarrow & \int_{\Omega} \int_{\mathbf{R}^{n m}} L\left(x, u_{0}(x), \cdot\right) d \nu_{x} d x \\
& =\int_{\Omega} L^{q c}\left(x, u_{0}(x), \nabla u_{0}(x)\right) d x=I^{q c}\left(u_{0}\right)
\end{aligned}
$$


By the corollary of Theorem 4.2 the identity

$$
L^{q c}(x, u, v)=\inf _{\phi \in C_{0}^{\infty}\left(\Omega ; \mathrm{R}^{m}\right)} \frac{1}{\operatorname{meas} \Omega} \int_{\Omega} L(x, u, v+\nabla \phi(y)) d y
$$

holds for a.a. $x \in \Omega$ and all $u \in \mathbf{R}^{m}$.

By Theorem 5.1 the functional $I^{q c}$ is lower semicontinuous: $\lim _{k \rightarrow \infty} I^{q c}\left(u_{k}\right) \geq I^{q c}\left(u_{0}\right)$ for $u_{k} \rightarrow u_{0}$ in $W^{1, p}\left(\Omega ; \mathbf{R}^{m}\right)$. It is obvious also that $I(u) \geq I^{q c}(u), u \in W^{1, p}(\Omega)$.

This completes the proof. QED

Remark 1. - The growth conditions from the theorem may be dropped if one considers more special class of integrands. Let $L: \Omega \times \mathbf{R}^{n m} \rightarrow \mathbf{R}$ be a Caratheodory integrand such that $0 \leq L(x, v) \leq A|v|^{p}+B, p \geq 1$. Then, the sequential weak lower semicontinuous envelope of the functional $I(u)$, defined as

$$
\tilde{I}(u)=\inf \left\{\liminf _{k \rightarrow \infty} I\left(u_{k}\right): u_{k} \rightarrow u \text { in } W^{1, p}\left(\Omega ; \mathbf{R}^{m}\right)\right\}
$$

is an integral functional with the integrand $L^{q c}$. This can be proved through approximation of the original functional by ones satisfying standard growth conditions.

Consider first the case of continuous $L$ and compact $\Omega$. As in the proof of Theorem 4.6 consider a family of auxiliary integrands $L_{\mu}$, where $L_{\mu}(x, v)=L(x, v)+\mu|v|^{2 p}, \mu>0$, and their quasiconvexifications $L_{\mu}^{q c}$, for which all conclusions of Theorem 1.2 hold. Hence $L_{1 / i}^{4 c}(i \in \mathbf{N})$ is a sequence of continuous functions quasiconvex in $v$ and decreasing to a function $\tilde{L}$ such that $0 \leq \tilde{L}(x, v) \leq A|v|^{p}+B$.

By the arguments from the proof of Theorem 4.6 (proposed for the case $p=1$ ) we obtain that $\tilde{L}$ is quasiconvex and continuous in $v$ and upper semicontinuous in $x$. Then $\tilde{L}$ is a Caratheodory integrand that implies existence of a sequence of compact subsets $\Omega_{k}$ of $\Omega$ such that $\operatorname{meas}\left(\Omega \backslash \Omega_{k}\right) \rightarrow 0$ as $k \rightarrow \infty$ and the restrictions of $\tilde{L}$ to $\Omega_{k} \times \mathbf{R}^{n m}$ are continuous. Note that by the Dini convergence theorem the sequence $L_{1 / i}^{q c}$ converges to $\tilde{L}$ locally uniformly in each compact subset of $\Omega_{k} \times \mathbf{R}^{n m}$.

For Caratheodory integrands $L$ and general $\Omega$ we may reduce the considerations to the particular case treated above. This proves that $\tilde{L}$ is always of Caratheodory type. The rest is a straightforward consequence of the construction. 
Remark 2. - It follows from the proof of Theorem 1.2 that $L^{q^{c}}$ is continuous for continuous and coercive $L$ (here coercivity means $A_{1}|v|^{p}+B_{1} \leq L \leq A_{2}|v|^{p}+B_{2}, A_{2} \geq A_{1}>0$ ). In the case of noncoercive $L$ the function $L^{q c}$ can have discontinuities. To construct a desired example notice that there exists a continuous integrand $L(x, v):[0,1] \times \mathbf{R} \rightarrow[0, \infty]$ such that $L(0, v) \geq v^{2}$ and $L(x, v)=0$ for $\left.\left.x \in\right] 0,1\right],|v| \geq M(x)$ (here $M(x) \rightarrow \infty$ as $x \rightarrow 0$ ). Then $L^{q c}(x, v)=0$ for $|x| \neq 0, L^{q c}(0, v) \geq v^{2}$.

Proof of the Theorem 1.4. - Without loss of generality we may suppose that $\left(u_{k}, \nabla u_{k}\right)$ generates a Young measure $\left(\delta_{u_{0}(x)} \otimes \nu_{x}\right)_{x \in \Omega}$ and the sequence $I\left(u_{k}\right)$ converges as $k \rightarrow \infty$. By Theorem 3.7

$$
\lim _{k \rightarrow \infty} I\left(u_{k}\right) \geq \int_{\Omega} \int_{\mathbf{R}^{n m}} L\left(x, u_{0}(x), \cdot\right) d \nu_{x} d x
$$

Moreover, equality holds if and only if the functions $L\left(x, u_{k}(x), \nabla u_{k}(x)\right)$ are equi-integrable. By the Localization principle $\nu_{x}$ is a gradient pYoung measure for a.a. $x \in \Omega$. Because $L\left(x, u_{0}(x), \cdot\right)$ is strictly closed p-quasiconvex at $\nabla u_{0}(x)$ for a.e. $x \in \Omega$ the inequality

$$
\int_{\mathbf{R}^{n m}} L\left(x, u_{0}(x), \cdot\right) d \nu_{x} \geq L\left(x, u_{0}(x), \nabla u_{0}(x)\right)
$$

holds for all such $x$, where the equality holds if and only if $\nu_{x}=\delta_{\nabla u_{0}(x)}$. Hence the convergence $I\left(u_{k}\right) \rightarrow I\left(u_{0}\right)$ holds if $u_{k} \rightarrow u_{0}$ in $W^{1,1}\left(\Omega ; \mathbf{R}^{m}\right)$ and the functions $L\left(\cdot, u_{k}(\cdot), \nabla u_{k}(\cdot)\right)$ are equi-integrable, cf. Theorem 3.7 and Proposition 3.8. This proves the first claim of the theorem.

To prove the second claim consider an increasing sequence of compact subsets $\Omega_{k}$ of the interior of $\Omega$ such that the restrictions of $u_{0}, \nabla u_{0}$ to $\Omega_{k}$ are continuous, the restrictions of $L$ to $\Omega_{k} \times \mathbf{R}^{m} \times \mathbf{R}^{n m}$ are continuous and meas $\left(\Omega \backslash \Omega_{k}\right) \rightarrow 0$ as $k \rightarrow \infty$. Let us establish first that either $L\left(x, u_{0}(x), \cdot\right)$ or $-L\left(x, u_{0}(x), \cdot\right)$ is quasiconvex at $\nabla u_{0}(x)$ for a.a. $x \in \Omega$. Otherwise there exists $k \in \mathbf{N}$, Lebesgue points $x_{1}, x_{2}$ of $\Omega_{k}$ and gradient $\mathbf{p}$-Young measures $\nu_{1}, \nu_{2}$ with the centers of mass at $\nabla u_{0}\left(x_{1}\right), \nabla u_{0}\left(x_{2}\right)$ respectively such that

$$
\begin{gathered}
\int_{\mathbf{R}^{n m}} L\left(x_{1}, u_{0}\left(x_{1}\right), \cdot\right) d \nu_{1}<L\left(x_{1}, u_{0}\left(x_{1}\right), \nabla u_{0}\left(x_{1}\right)\right)-\epsilon, \\
\int_{\mathbf{R}^{n m}} L\left(x_{2}, u_{0}\left(x_{2}\right), \cdot\right) d \nu_{2}>L\left(x_{2}, u_{0}\left(x_{2}\right), \nabla u_{0}\left(x_{2}\right)\right)+\epsilon, \epsilon>0 .
\end{gathered}
$$


We can isolate neighborhoods $V_{1}, V_{2}$ of $x_{1}, x_{2}$ in $\Omega_{k}$ such that

$$
\begin{gathered}
\int_{\mathbf{R}^{n m}} L\left(x, u_{0}(x), \cdot\right) d\left(\nu^{1} \diamond \nabla u_{0}(x)\right)<L\left(x, u_{0}(x), \nabla u_{0}(x)\right)-\epsilon, \quad x \in V_{1}, \\
\int_{\mathbf{R}^{n m}} L\left(x, u_{0}(x), \cdot\right) d\left(\nu^{2} \diamond \nabla u_{0}(x)\right)>L\left(x, u_{0}(x), \nabla u_{0}(x)\right)+\epsilon, \quad x \in V_{2}, \\
\int_{V_{1} \cup V_{2}} L\left(x, u_{0}(x), \nabla u_{0}(x)\right) d x= \\
\int_{V_{1}}\left\{\int_{\mathbf{R}^{n m}} L\left(x, u_{0}(x), \cdot\right) d\left(\nu^{1} \diamond \nabla u_{0}(x)\right)\right\} d x \\
+\int_{V_{2}}\left\{\int_{\mathbf{R}^{n m}} L\left(x, u_{0}(x), \cdot\right) d\left(\nu^{2} \diamond \nabla u_{0}(x)\right)\right\} d x .
\end{gathered}
$$

By Theorem 4.3 a Young measure $\left(\nu_{x}\right)_{x \in \Omega}$, which equals $\nu^{1} \diamond \nabla u_{0}$ on $V_{1}, \nu^{2} \diamond \nabla u_{0}$ on $V_{2}$, and $\delta_{\nabla u_{0}(x)}$ for other $x \in \Omega$, is a nontrivial gradient $\mathrm{p}$-Young measure with the centers of mass at $\nabla u_{0}(x), x \in \Omega$. Moreover, the weak-strong convergence property fails for a sequence associated with this measure (cf. Proposition 4.4). This contradiction proves that either $L\left(x, u_{0}(x), \cdot\right)$ or $-L\left(x, u_{0}(x), \cdot\right)$ is quasiconvex at $\nabla u_{0}(x)$ for a.a. $x \in \Omega$.

Therefore we may assume without loss of generality that $L\left(x, u_{0}(x), \cdot\right)$ is quasiconvex at $\nabla u_{0}(x)$ for a.e. $x \in \Omega$.

For a fixed $k \in \mathbf{N}$ consider the set $K_{l}$ ( $l$ is a natural number) consisting of all $x \in \Omega_{k}$ such that there exists a gradient $\mathrm{p}$-Young measure $\nu$ with the center of mass at $\nabla u_{0}(x)$, for which the following holds:

$$
\begin{gathered}
\int_{\mathbf{R}^{n m}}\left(1+|\cdot|^{p}\right) d \nu<k, \\
\int_{\mathbf{R}^{n m}} L\left(x, u_{0}(x), \cdot\right) d \nu<L\left(x, u_{0}(x), \nabla u_{0}(x)\right)+1 / l, \\
\nu\left(\left\{v \in \mathbf{R}^{n}: 1 / k \leq\left|v-\nabla u_{0}(x)\right| \leq k\right\}\right) \geq 1 / k .
\end{gathered}
$$

We will prove that meas $K_{l} \rightarrow 0$ as $l \rightarrow \infty$ by contradiction. This fact is enough to establish the second claim of the theorem.

The sets $K_{l}$ are open in $\Omega_{k}$. Actually, if (5.1)-(5.3) hold for a measure $\nu \in G M_{\nu}\left(\nabla u_{0}\left(x_{0}\right)\right)$ and a point $x_{0} \in K_{l}$ then the same holds for any $z \in \Omega_{k}$ sufficiently close to $x_{0}$ with $\nu \diamond \nabla u_{0}(z)$ instead of $\nu$.

Since we have assumed that $\lim _{l \rightarrow \infty}$ meas $K_{l}>2 \delta_{0}>0$ and $K_{l+1} \subset K_{l}$ we obtain meas $\left(\cap_{l} K_{l}\right) \geq 2 \delta_{0}$. Let $K \subset \cap_{l} K_{l}$ be a compact set such that $\operatorname{meas} K>\delta_{0}$. 
Fix $l \in \mathbf{N}$. Then for each $x \in K$ we can find a ball $B$ with the center at this point and such that for each point $z \in B \cap K$ inequalities (5.1)-(5.3) hold with $\nu \diamond \nabla u_{0}(z)$, where $\nu \in G M_{p}\left(\nabla u_{0}(x)\right)$. Let $B\left(x_{i}, \epsilon_{i}\right)\left(i=1, \ldots, l^{\prime}\right)$ be a finite cover of $K$ by such balls and let $\nu\left(x_{i}\right) \in G M_{p}\left(\nabla u_{0}\left(x_{i}\right)\right)$ be measures associated with the centres of these balls. Let $\Omega_{1}^{l}=B\left(x_{1}, \epsilon_{1}\right) \cap K$, $\Omega_{2}^{l}=\left(B\left(x_{2}, \epsilon_{2}\right) \backslash \bar{B}\left(x_{1}, \epsilon_{1}\right)\right) \cap K$. For other $i \geq 2$ define $\Omega_{i+1}^{l}$ as $\left(B\left(x_{i+1}, \epsilon_{i+1}\right) \backslash \cup_{j=1}^{i} \bar{B}\left(x_{j}, \epsilon_{j}\right)\right) \cap K$. Consider also compact subsets $\tilde{\Omega}_{i}^{l}$ of $\Omega_{i}^{l}$ such that meas $\left(\cup_{i} \tilde{\Omega}_{i}^{l}\right)=\delta_{0}$.

The measure $\left(\nu_{x}^{l}\right)_{x \in \Omega}$, which equals $\nu\left(x_{i}\right) \diamond \nabla u_{0}(x)$ for $x \in \tilde{\Omega}_{i}^{l}$ $\left(i=1, \ldots, l^{\prime}\right), \delta_{\nabla u_{0}(x)}$ - otherwise, is a gradient p-Young measure. Actually, if we replace $\tilde{\Omega}_{i}^{l}$ by disjoint open sets then the claim follows from Lemma 2.2 and Proposition 4.4. By Proposition 2.3 we can approximate $\tilde{\Omega}_{i}$ by such open sets, that leads to the desired result again through Lemma 2.2.

By the compactness theorem there exists a subsequence $\left(\nu_{x}^{l}\right)_{x \in \Omega}$ (not relabeled) converging weakly* in $L_{w}\left(\Omega ; K_{1}\right)$ to $\left(\nu_{x}\right)_{x \in \Omega}$. Because (5.3) holds for any $x \in \cup_{i} \tilde{\Omega}_{i}^{l}$ (with $\nu_{x}^{l}$ instead of $\nu$ ) and meas $\left(\cup_{i} \tilde{\Omega}_{i}^{l}\right) \geq \delta_{0}>0$ for each $l \in \mathbf{N}$ the Young measure $\left(\nu_{x}\right)_{x \in \Omega}$ is not trivial.

For each $l \in \mathbf{N}$ there exists a sequence $u_{j}^{l} \in u_{0}+C_{0}^{\infty}\left(\Omega ; \mathbf{R}^{m}\right), j \in \mathbf{N}$, generating $\left(\nu_{x}^{l}\right)_{x \in \Omega}$ as a gradient p-Young measure (cf. Proposition 4.4). We can isolate a sequence $u_{j(l)}^{l}, l \in \mathbf{N}$, such that

$$
\begin{gathered}
\left.\left(\delta_{\nabla u_{j(l)}^{l}(x)}\right)_{x \in \Omega} \vec{*}^{*}\left(\nu_{x}\right)_{x \in \Omega}, \int_{\Omega}\left(1+\left|\nabla u_{j(l)}^{l}(x)\right|^{p}\right) d x<k \text { (because of }(5.1)\right), \\
\left.\left|I\left(u_{j(l)}^{l}\right)-I\left(u_{0}\right)\right|<2 \operatorname{meas} \Omega / l \text { (because of }(5.2)\right) .
\end{gathered}
$$

Then $u_{j(l)}^{l}-u_{0}$ in $W^{1, p}\left(\Omega ; \mathbf{R}^{m}\right)$ and the weak-strong convergence property fails along the sequence $u_{j(l)}^{l}$ in view of nontriviality of $\left(\nu_{x}\right)_{x \in \Omega}$ (cf. Proposition 3.8).

This contradiction proves that meas $K_{l} \rightarrow 0$ as $l \rightarrow \infty$, and, as a consequence, that the set of all $x \in \Omega_{k}$ for which $L\left(x, u_{u}(x), \cdot\right)$ is not strictly p-quasiconvex at $\nabla u_{0}(x)$ has zero measure.

The proof of the theorem is completed. QED

Remark. - If the function $L\left(x, u_{0}(x), \cdot\right)$ is quasiconvex at $\nabla u_{0}(x)$ for a.e. $x \in \Omega$ and $L$ is bounded from below a simpler proof for the second part of the theorem is available (see $[45, \S 3]$ ). Moreover, in this case it is enough to restrict considerations to sequences $u_{k}$ with equi-integrable $\left|\nabla u_{k}\right|^{p}$.

In this case we may consider the set $\Omega_{j, k}$ consisting of all $x \in \Omega_{k}$, for each of which there exists a gradient p-Young measure $\nu$ with the center 
of mass at $\nabla u_{0}(x)$ and such that

$$
\begin{gathered}
\int_{\mathbf{R}^{n m}}\left(1+|\cdot|^{p}\right) d \nu \leq j, \\
\int_{\mathbf{R}^{n m}} L\left(x, u_{0}(x), \cdot\right) d \nu=L\left(x, u_{0}(x), \nabla u_{0}(x)\right), \\
\nu\left(B\left(\nabla u_{0}(x), 1 / j\right)\right) \leq 1-1 / j .
\end{gathered}
$$

This defines a multivalued mapping $V: \Omega_{j, k} \rightarrow 2^{\mathcal{M}\left(\mathbf{R}^{n m}\right)}$, where $V(\cdot)$ consists of elements of $G M_{p}(\cdot)$ satisfying (5.4)-(5.6). Because of boundedness of $L$ from below it is not hard to prove that this mapping is closed and upper semicontinuous in $\rho$ metric (see $\S 3$ of [45]). By Theorems 3.3 and 3.5 there exists a selection $\left(\mu_{x}\right)_{x \in \Omega_{j, k}} \in L_{w}\left(\Omega_{j, k} ; K_{1}\right)$, which automatically satisfies conditions (i)-(iii) of Theorem 4.3. Let $\nu_{x}=\mu_{x}$ for $x \in \Omega_{j, k}$, and $\nu_{x}=\delta_{\nabla u_{0}(x)}$ for $x \in \Omega \backslash \Omega_{j, k}$. Then $\left(\nu_{x}\right)_{x \in \Omega}$ is a gradient p-Young measure by Theorem 4.3. Hence, if meas $\Omega_{j, k}>0$ then the weakstrong convergence property fails along the sequence of Sobolev functions associated with $\left(\nu_{x}\right)_{x \in \Omega}$. This proves that meas $\Omega_{j, k}=0$ for any $j, k \in N$. Therefore $L\left(x, u_{0}(x), \cdot\right)$ is strictly p-quasiconvex at $\nabla u_{0}(x)$ for a.e. $x \in \Omega$.

In the situation of Theorem 1.4 one may consider analogous multi-valued mapping $V$, but growth conditions do not suffice to prove closedness and upper semicontinuity of $V$ in $\rho$ metric. These properties hold with respect to convergence in the metric $\tilde{\rho}(\nu, \mu):=\rho(\nu, \mu)+\left|\left\langle 1+|\cdot|^{p} ; \nu\right\rangle-\left\langle 1+|\cdot|^{p} ; \mu\right\rangle\right|$, but both $V$ and $G M_{p}(A)$ are not complete in this metric. Probably more subtle theorems on measurable selections (see e.g. [12]) can be helpful here in order to utilize selection arguments and, as a consequence, to restrict the class of sequences $u_{k}$ to ones with equi-integrable $\left|\nabla u_{k}\right|^{p}$.

\section{ACKNOWLEDGMENTS}

It is great pleasure to thank Prof.S.Müller for the help of various type during my visit in Freiburgh in 1995. I am grateful to him and to unknown referees for criticism of a preliminary version of this paper, which let me much improve its presentation and forced me to make proofs more detailed. I thank Prof. Dal Maso for interest to this work and for the support.

After submission of the first version [48] of this paper a book [40] appeared. The author of [40] also suggests to use Young measure techniques to prove the relaxation theorem, see $[40$, p.65]. He proposes to use the formula

$$
L^{q c}(A)=\inf _{\nu \in G M^{p}(A)}\langle L ; \nu\rangle
$$

Vol. 16, $n^{\circ}$ 6-1999. 
which was first established in [29], to construct a gradient p-Young measure $\left(\nu_{x}\right)_{x \in \Omega}$ with the property

$$
\left\langle L\left(x, u_{0}(x), \cdot\right) ; \nu_{x}\right\rangle=L^{q x}\left(x, u_{0}(x), D u_{0}(x)\right) \text { for a.a. } x \in \Omega .
$$

One of the aims of this our work was to develop Young measure calculus up to the level which let one make similar arguments rigorous.

\section{REFERENCES}

[1] E. ACERbI, N. FusCo, Semicontinuity problems in the calculus of variations. Arch. Rat. Mech. Anal., Vol. 86, 1984, pp. 125-145.

[2] E. ACERbi, G. Dal Maso, New lower semicontinuity results for polyconvex integrals, Calc.Var. \& PDE 2 (1994), no. 3, pp. 329-371.

[3] E.J. BALDER, A general approach to lower semicontinuity and lower closure in optimal control theory, SIAM J. Control and Optimization, Vol. 22, 1984, pp. 570-598

[4] J.M. BALL, Convexity conditions and existence theorems in nonlinear elasticity, Arch. Rat. Mech. Anal., Vol. 63, 1978, pp. 337-403.

[5] J.M. BALL, A version of the fundamental theorem for Young measures, in: PDE's and Continuum Models of Phase Transitions, M.Rascle, D.Serre, M.Slemrod, eds., Lecture Notes in Physics 344, Springer-Verlag (1989), pp. 207-215.

[6] G. Bouchitte, I. Fonseca, J. MaLY, The Effective Bulk Energy of the Relaxed Energy of Multiple Integrals Below the Growth Exponent, Proc. Royal Soc. Edinb. Sect A Vol. 128, 1998, pp. 463-479.

[7] J.M. BALL, F. MURAT, $W^{1, p}$-quasiconvexity and variational problems for multiple integrals, J. Funct. Anal., Vol. 58, 1984, pp. 225-253.

[8] H. BeRLIOCCHI, J.M. LASRY, Intégrandes normales et mesures paramétrées en calcul des variations, Bull. Soc. Math. France, Vol. 101, 1973, pp.129-184.

[9] J.M. BALL, K. ZHANG, Lower semicontinuity of multiple integrals and the bitting lemma, Proc. Roy. Soc. Edinburgh Sect A., Vol. 114, 1990, pp. 367-379.

[10] G. BuTTAzzo, Lower Semicontinuity, Relaxation and Integral Representation in the Calculus of Variations, Pitman Research Notes in Math. Series 207, 1989.

[11] P. Celada, G. G. Dal Maso, Further remarks on the lower semicontinuity of polyconvex integrals, Ann. Inst. H. Poincaré Anal. Non. Linéaire, Vol. 11, 1994, no. 6, pp. 661-691.

[12] C. CASTAing, M. VAladiER, Convex analysis and measurable multifunctions, Lecture Notes in Mathematics 580, Springer-Verlag, Berlin-New York, 1977.

[13] A. Cellina, S. ZagatT, A version of Olech's lemma in a problem of the Calculus of Variations, SIAM J. Control and Optimization, Vol. 32, 1994, pp. 1114-1127.

[14] B. DACOROGNA, Weak continuity and weak lower semicontinuity of nonlinear problems, Lecture Notes in Math. 922, Springer-Verlag, 1982.

[15] B. Dacorogna, Direct methods in the Calculus of Variations. Springer-Verlag, 1989.

[16] G. DAL MASO, C. SBordone, Weak lower semicontinuity of polyconvex integrals: a borderline case. Math. Z., Vol. 218, 1995, no. 4, pp. 603-609.

[17] R.E. Edwards, Functional Analysis, Holt, Rinehart and Winston, 1965.

[18] I. Ekeland, R. Temam, Convex analysis and variational problems. North-Holland, 1976.

[19] L.C. Evans, R.F.GariEPY, Some remarks on quasiconvexity and strong convergence, Proc. Roy. Soc. Edin. Sect. A, Vol. 106, 1987, pp. 53-61.

[20] L.C.Evans, Weak convergence methods for nonlinear partial differential equations. AMS, 1990.

[21] N.FusCo, J. HUTCHINSON, A direct proof for lower semicontinuity of polyconvex functionals, Manuscripta Math., Vol. 87, 1995, no.1, pp. 35-50. 
[22] I. FonsecA, J. Maly, Relaxation of Multiple Integrals in Sobolev Spaces Below the Growth Exponent for the Energy Density, Ann. Inst. Henri Poincaré Anal. Non. Linéaire, Vol. 14, 1997, no. 3, pp. 309-338.

[23] I. Fonseca, S. Müller, P. Pedregal, Analysis of Concentration and Oscillation Effects Generated by Gradients. To appear in SIAM J. Math. Anal.

[24] M. Giaquinta, G. Modica, J. SoúcEK, Remarks on lower semicontinuity of quasiconvex integrals. NoDEA Nonlinear Differential Equations Appl., Vol. 2, 1995, no. 4, pp. 573-588.

[25] T. IWANIEC, C. SBORDONE, On the integrability of the jacobian under minimal hypotheses, Arch. Rat. Mech. Anal., Vol. 119, 1992, pp. 129-143..

[26] R. Jordan, D. Kinderlehrer, An extended variational principle, Partial differential equations and applications, M. Dekker, 1996, pp. 187-200.

[27] D. Kinderlehrer, P. Pedregal, Characterization of Young measures generated by gradients, Arch. Rat. Mech. Anal., Vol. 115, 1991, pp. 329-365.

[28] D. Kinderlehrer. P. Pedregal, Weak convergence of integrands and the Young measure representation, SIAM J. Math. Anal., Vol. 23, 1992, pp. 1-19.

[29] D. Kinderlehrer, P. Pedregal, Gradient Young measures generated by sequences in Sobolev spaces, J. Geom. Anal., Vol. 4, 1994, no 1, pp. 59-90.

[30] J. KRISTENSEN, Finite functionals and Young measures generated by gradients of Sobolev functions, Ph. D. Thesis, Technical University of Denmark, Kyngby, August 1994.

[31] K. Kuratowski, Ryll-Nardzewski, A general theorem of selectors, Bull. Acad. Polon. Sci., Vol. XIII, 1966, no. 6, pp. 397-403.

[32] P. Marcellini, Approximation of quasiconvex functions, and lower semicontinuity of multiple integrals, Manuscripta Math., Vol. 51, 1985, pp. 1-28.

[33] P. Marcellini, On the definition and the lower semicontinuity of certain quasiconvex integrals, Ann. Inst. H. Poincaré, Analyse non Linéaire, Vol. 3, 1986, pp. 391-409.

[34] J. Maly, Weak lower semicontinuity of polyconvex integrals., Proc. Roy. Soc. Edinburgh., Sect A, Vol. 123, 1993, no.4, pp. 681-691.

[35] J. MALY, Lower semicontinuity of quasiconvex integrals, Manuscripta Math., Vol, 85 , 1994, no.3-4, pp. 419-428.

[36] C.B. MorREy, Quasi-convexity and the lower semicontinuity of multiple integrals, Pasific J. Math., Vol. 2, 1952, pp. 25-53

[37] C.B. Morrey, Multiple integrals in the Calculus of Variations. Springer-Verlag, 1966.

[38] P. Marcellini, C. Sbordone, Semicontinuity problems in the Calculus of Variations, Nonlinear Anal., Vol. 4, 1980, pp. 241-257.

[39] P. Pedregal, Jensen's inequality in the calculus of variations, Differential and Integral Equations, Vol. 7, 1994, pp. 57-72.

[40] P. Pedregal, Parametrized measures and variational principles. Birkhäuser, 1997.

[41] Y.G. RESHETNYAK, General theorems on semicontinuity and convergence with a functionals, Sibirsk. Math. Z., Vol. 8, 1967, pp. 1051-1069.

[42] Y.G. Reshetnyak, Space mappings with bounded distortion. Translations of Mathematical Monographs, 73, Amcrican Mathematical Society. Providence, RI, 1989.

[43] Y.G. RESHETNYAK, A convergence theorem for functionals on additive vector-valued set functions, Sibirsk. Mat. Z., Vol. 2, 1961, pp. 115-126.

[44] M. Sychev, Necessary and sufficient conditions in theorems of lower semicontinuity and convergence with a functional, Russ. Acad, Sci. Sb. Math., Vol. 186, 1995, pp. 847-878.

[45] M. SYCHEV, Young measure approach to characterization of behavior of integral functionals on weakly convergent sequences by means of their integrands, Ann. Inst. H. Poincaré Anal. Non. Linéaire, Vol. 15, 1998, no. 6.

[46] M. SYCHEv, Young measures as measurable functions (in preparation).

[47] M. Sychev, Characterization of homogeneous gradient Young measures in the case of arbitrary integrands. Report No. 98-216, May 98, Carnegie Mellon University.

[48] M. SYchev, New approach to Young measure theory, relaxation and convergence in energy. SISSA preprint 44/97/M, March 1997, Triest.

Vol. 16, n 6-1999. 
[49] L. TARTAR, Compensated compactness and applications to partial differential equations, in Nonlinear analysis and mechanics: Heriot-Watt Symposium, Vol. IV, Pitman Research Notes in Mathematics, Vol. 39, 1979, pp. 136-212.

[50] L. TARTAR, The compensated compactness method applied to systems of conservations laws, in Systems of Nonlinear Partial Differential Equations, J. M. Ball ed., NATO ASI Series, Vol. CIII, Reidel, 1982.

[5I] M. ValadiER, Young measures, in Methods of Nonconvex Analysis, ed. A. Cellina, Lecture Notes in Math., Vol. 1446, 1990, pp. 152-188.

[52] G.V. Vasilenko, Convergence with a functional, Sibirsk. Math. Z., Vol. 27, 1986, no. 1, pp. 26-34.

[53] A. Visintin, Strong convergence results related to strict convexity, Comm. Partial Differential Equations, Vol. 9, 1984 , pp. 439-466.

[54] L.C. Young, Lectures on the Calculus of Variations and Optimal Control Theory. Saunders, 1969 (reprinted by Chelsea 1980).

[55] L.C. Young, Generalized curves and the existence of an attained absolute minimum in the Calculus of Variations, Comptes Rendus de la Société des Sciences et des Lettres de Varsovie, classe III, Vol. 30, 1937, pp. 212-234.

[56] W.P.ZIEMER, Weakly differentiable functions. Springer-Verlag, New-York, 1989.

(Manuscript received April 2, 1997;

revised June 6, 1998.) 